\title{
Asociación entre creencias implícitas acerca de la inteligencia y el rendimiento académico en escolares de 10 a 14 años de Arequipa metropolitana
}

The Association Between Implicit Theories of Intelligence and Academic

Achievement in Children Between 10 to 14 Years of Age

from Metropolitan Arequipa

Joselyn Mayeli Melo Peña

Universidad Católica San Pablo, Arequipa, Perú.

(iD) https://orcid.org/oooo-ooo1-6911-4514

Rosario Carolina Salcedo Soto

Universidad Católica San Pablo, Arequipa, Perú

(iD https://orcid.org/oooo-0oo2-6468-5016

Correspondencia: rosario.salcedo@ucsp.edu.pe

\section{Resumen}

La presente investigación tiene por objetivo determinar la asociación entre las creencias implícitas acerca de la inteligencia de los escolares con el desempeño escolar de estudiantes de colegios nacionales y particulares del área metropolitana de Arequipa del 5 to y 6 to grado de primaria y 1er año de secundaria. Corresponde a un diseño no experimental, transversal y correlacional. Se encuestaron a 434 estudiantes de colegios públicos y privados de Arequipa Metropolitana. Se administró la Escala de Teorías Implícitas, traducida y adaptada a la población colombiana y al contexto peruano. Los principales resultados evidencian que los colegios privados presentaron puntajes más altos de mentalidad fija, así como un mejor rendimiento. Se concluye que el rendimiento académico bajo puede verse influenciado por la creencia de la inteligencia incremental, además de no contar con el apoyo en los estudios y entrega de recursos académicos.

Palabras clave: Rendimiento académico, inteligencia, creencia fija, creencia incremental.

\section{Abstract}

The present study was conducted to determine if there was an association between implicit theories and academic achievement in schoolchildren in the 
5th and 6th grade of primary school and the ist grade of secondary school. A no experimental, transversal, correlational design was used for this study. 434 students in public and private educational institutions in Metropolitan Arequipa were surveyed. The Implicit Theory Scale was used. This scale was translated and adapted for a Colombian population and was adapted to the Peruvian context. Results indicated that private schools had higher fixed-mind scores, as well as better performance. There was a significant association between family support and academic performance. It was concluded that low academic performance could be influenced by a growth mentality, not having support in their studies, and delivery of academic resources.

Keywords: Academic performance, intelligence, fixed belief, incremental belief.

\section{Introducción}

De acuerdo con los resultados del PISA 2015 (Programme for International Student Assessment) llevado a cabo por Organización para la Cooperación y Desarrollo Económico; nuestro país se encuentra dentro de los diez países con rendimiento académico más bajos en las áreas de matemática, lectura y ciencia a nivel mundial. Aproximadamente tres de cada cuatro alumnos no aprobaron matemáticas, un promedio del $70 \%$ de las evaluaciones en ciencias fue anulada y el $60 \%$ demostró que no entendía lo que leía (BBC, 2016). Si bien los resultados de PISA 2018 dieron a conocer que el Perú ocupa el puesto 64 de 77 países, donde se observan ciertas mejorías, se tienen resultados similares a la prueba del 2015, debido a que los alumnos evaluados continuamos dentro de los últimos puestos a nivel de los países latinoamericanos (Gestión, 2019). A partir del Informe Nacional de Evaluación Censal de Estudiantes 2018 (ECE) que realizó el Ministerio de Educación del Perú, se conoce que la mayoría de los escolares del año 2019 se encontraron en un nivel de logro elemental o parcial.
Hace algunos años Carol Dweck (2007) planteó una nueva perspectiva que intenta abordar la problemática ligada al desempeño escolar haciendo referencia a las creencias implícitas que tienen los alumnos respecto a su propia inteligencia, a lo que se denomina teoría implícita de la inteligencia. Este constructo está dispuesto en un continuo que va desde la inteligencia como fija hasta la inteligencia como incremental. El primero considera a la capacidad cognitiva como estable e inmutable a lo largo del tiempo. Por tanto, personas con este tipo de creencias sobre la inteligencia tienden a atribuir un mal desempeño académico a una pobre inteligencia, por lo cual invierten un menor esfuerzo por cambiar su situación académica. De este modo se observa una especie de auto profecía cumplida. Por el contrario, la creencia incremental refiere que la inteligencia es maleabley es posible mejorarla con esfuerzo; por tanto, esto favorece al desempeño académico.

De acuerdo a Dweck (2007) es posible cambiar estas creencias ya que serían 
disposiciones mentales, y de ese modo se puede ayudar en el desempeño académico de los escolares. Las teorías implícitas de Dweck (2007) han sido estudiadas en países como China (Wang \& Fei-YinNg, 2012), Australia (Tarbetsky \& Martín, 2016), Francia (Lammel \& Guillén, 2011), España (Carpintero et al., 2003), México (Lammel \& Guillén (2011) y Colombia (Galvis et al., 2010), mas no en Perú.

Por ello, en la presente investigación se tiene por objetivos:1) identificar el tipo de creencias implícitas acerca de la inteligencia que tienen los estudiantes del 5to y 6to

\section{Inteligencia}

\section{Definiciones de Inteligencia}

Alfred Binet definió la inteligencia señalando que es una capacidad fundamental que tiene efectos importantes para la vida práctica. Sin embargo, sostuvo que los alumnos requieren aprender de otros aspectos que no se tomaron en cuenta en la prueba de inteligencia que crea con Simon, como es el caso de la atención, la voluntad y el carácter; los cuales se reflejan en la docilidad, la disciplina, los hábitos regulares y especialmente en la continuidad de esfuerzo para aprender (Binet \& Simon, 1916). Por otro lado, Charles Spearman (1904) se refirió a la inteligencia como un conjunto de habilidades cognitivas que se relacionan entre sí, sin que ninguna de ellas sea un factor principal de la misma. Por ende, se enfocó en la generalización de la inteligencia llamada factor G (Fineburg, 2009). grado de primaria y 1er año de secundaria del área metropolitana de Arequipa, 2) describir el nivel del desempeño escolar de los estudiantes del 5 to y 6 to grado de primaria y $1 e r$ año de secundaria del área metropolitana de Arequipa y 3 ) determinar las diferencias de nivel académico, nivel socioeconómico, entorno familiar, sexo y tipo de colegio (particular o nacional) en los tipos de creencias implícitas acerca de la inteligencia y el desempeño académico de estudiantes 5 to y 6 to grado de primaria y er año de secundaria del área metropolitana de Arequipa.

Mientras que Catell y Horn (1967) expresaron que la inteligencia está compuesta por habilidades fluidas que guían la habilidad individual de un sujeto, a razonar y actuar con rapidez, resolver nuevos problemas y codificar memorias de corto plazo. Asimismo, sostuvieron una diferencia entre inteligencia fluida y cristalizada. La inteligencia fluida está enraizada en la eficiencia fisiológica (Horn, 1965). El otro factor, llamado inteligencia cristalizada, provienen del aprendizaje y la influencia de la cultura, y se plasma en tests de conocimiento, información general, el uso del lenguaje y una diversidad de habilidades que se adquieren (Horn \& Catell, 1967), ero ambas dependen una de la otra (Horn \& Catell, 1966).

También, Piaget (1969) planteó una visión de la inteligencia centrada en cómo se obtiene el conocimiento en la medida que uno va desarrollándose, y que 
evolucionaría a través de etapas Su contribución permitió modificar el rumbo de la investigación dedicada al desarrollo del niño, combatiendo la visión de ser un organismo pasivo, condicionado y moldeado por el entorno.

A su vez, Vigotsky (1979, citado por Arias, 2013) refirió que la inteligencia si bien precisa del factor hereditario también lo depende de las influencias culturales, siendo estas últimas las más relevantes, ya que permite la modificación de la inteligencia, la cual toma la dirección que cada cultura le impone. Por ende la inteligencia puede acrecentarsey también decrecer, de manera que la persona al recibir estímulos beneficiosos de su entorno, enriquece su pensamiento y acorta la distancia entre la zona de desarrollo real (ZDr) y la zona de desarrollo potencial (ZDp), permitiendo así, el desarrollo de la inteligencia. Por otro lado, Gardner (1987, citado por Macías) señala que la inteligencia implica la habilidad para resolver un problema o para elaborar productos que son importantes en un contexto cultural. Por tanto, este enfoque se vio dirigido a rescatar también el aspecto cultural (Berríos, 2018), buscando conocer cómo una persona manifiesta su inteligencia, de modo que se postula la existencia de siete inteligencias, teoría que ha generado diversas críticas (Arias, 2014).

En lo que respecta a la presente investigación tomaremos en cuenta la definición de inteligencia según Dweck, quien refiere, que es una suma de capacidades mentales que orientan las emociones, los pensamientos y las acciones para conocer, comprender y hallar soluciones a los problemas habituales de forma táctica y eficaz (Fandiño, 2008).

\section{Teorías implícitas acerca de la inteligencia}

\section{Definición de las Teorías Implícitas}

Carol Dweck, docente de psicología en la Universidad de Stanford, sostiene que existen tres creencias que paralizan el desarrollo del intelecto: la primera es considerar la inteligencia como invariable, la segunda es respecto a medir el potencial a raíz del resultado conseguido en un momento dado, y la tercera creencia es sobre las personas que no requieren del esfuerzo para conseguir logros como el éxito académico puesto que poseen talentos innatos (Dweck, 1999). En efecto, las creencias de la inteligencia suponen que las acciones del individuo dependen de construcciones mentales no conscientes y tienen una vinculación afectiva, por lo cual denomino a esta propuesta como teoría implícita de la inteligencia. No obstante al estar inducidas por las experiencias y construcciones personales pueden cambiar como también adaptarse ante situaciones nuevas, perteneciendo a un dominio en particular y dependen de que sean reconocidas o no (Bollousa, 2014). En el ámbito académico, Dweck (2007) encontró una relación positiva entre el tipo de creencias acerca de la inteligencia con los resultados académicos de los estudiantes. En síntesis, según la autora la inteligencia es maleable y las creencias que tiene la persona sobre la inteligencia atañen en su rendimiento académico. 


\section{Tipos de creencias según la teoría implícita acerca de la inteligencia}

Carol Dweck desde sus inicios como investigadora tuvo un gran interés por conocer cómo las personas lidian con el fracaso, por lo cual se dedicó a hacer diversas investigaciones en el área educativa (Dweck, 2017). En efecto, Dweck (2007) detecto que las creencias en función a la inteligencia se clasificarían en: la creencia fija y la creencia de crecimiento. En cuanto a la creencia fija, se hace referencia a aquellas personas que tienen la idea de que su inteligencia no es modificable, tienden a evitar las dificultades que se les presentan, ya que son consideradas como aspectos externos a la persona, por ende siendo el esfuerzo y la práctica ineficientes se dan por vencidos con facilidad, y por tanto, en el plano académico, no se toman acciones para revertir la situación (Dweck, 2007). Es conveniente señalar que la evitación del esfuerzo sucede por dos motivos: creer que el logro sin esfuerzo es propia de genios, y porque muchas veces se encuentran justificaciones para no tomar riesgos que suponen enfrentar dificultades (Dweck, 2017). Por tanto, muchas veces los estudiantes tienden a enfocarse en lo negativo, creando identidad personal negativa en el plano académico. Así, cuando las personas con creencia fija dejan de sentirse inteligentes o talentosos en algún aspecto, simplemente pierden el interés en ello. Asimismo, en el plano de las interacciones personales, buscan relacionarse con personas que no les den a conocer sus características negativas, procurando mostrarse superiores en vez de buscar su propio desarrollo (Carpintero, et al.,
2003). Esto les genera también emociones negativas por sentir temor de perder las valoraciones positivas que se le atribuyen.

En cuanto a la creencia de crecimiento, la persona considera la posibilidad de mejora en sus habilidades para adquirir nuevos conocimientos, por lo que orienta sus metas al aprendizaje, lo cual significa asumiry superar las dificultades a lo largo del tiempo a través del esfuerzo (Gayón, 2010). De manera que se procura tomar el control de la situación, implementando buenas estrategias y recibiendo ayuda de los demás durante el proceso (Dweck, 2017). Por ende, tienen una interpretación resiliente de los desafíos, tanto académicos como de otro tipo, teniendo en cuenta que la resiliencia implica la respuesta emocional frente a las dificultades académicas o sociales, de forma positiva y beneficiosa para el desarrollo de la persona (Yeager \& Dweck, 2012). Por tanto, al estar interesados en mejorar y enfocarse en la lucha por superar las dificultades, desarrollan un mejor conocimiento de sus habilidades y capacidades (Dweck, 2017), yvan en búsqueda de interacciones que le permitan incrementar sus conocimientos, tomando las críticas como oportunidades de mejora (Carpintero et al., 2003).

Es importante señalar que la autora de la propuesta de la teoría implícita de la inteligencia, reconoce que hay factores que promueven que una persona pueda desarrollarse intelectualmente de forma positiva, como son los estímulos parentales y una buena educación (Dweck, 2017). Se tiene que señalar que en ambas creencias se pueden experimentan 
emociones negativas, pero la diferencia radica en cómo las interpretan según la confianza en sí mismo. Las personas con creencia fija se valoran en medida de las emociones negativas y muestran confianza sólo cuando nada malo les acontece (Dweck, 2017).

\section{¿Se puede tener ambas mentalidades?}

Con motivo de distinguir ambas mentalidades, se han presentado como alternativas opuestas, sin embargo, en la mente humana estas dos mentalidades o creencias, pueden coexistir en una misma persona, a pesar de que esto pueda parecer contradictorio o inconsistente (Dweck et al, 1996). Es posible que una de estas mentalidades se manifieste con mayor intensidad, o que una u otra mentalidad, se presenten en situaciones o áreas particulares (Dweck, 2017). Asimismo, es crucial comprender que el contexto y las experiencias del día a día de la persona, da lugar muchas veces una fusión de creencias fijas y de crecimiento (Bollousa, 2014).

\section{Desarrollo de las creencias acerca de la inteligencia del niño}

El proceso de transición de primaria a secundaria es un punto crítico para el desarrollo de los escolares, en el cual oscilan entre las edades de 10 a 14 años de edad. Si bien la escuela primaria es un entorno que brinda apoyo y contrarresta las consecuencias de las creencias fijas de la inteligencia, posteriormente, en la escuela secundaria, tienen lugar cambios académicos que pueden ser generar desafíos muy demandantes para los estudiantes. Además, se marca el inicio de la adolescencia, que es una etapa de cambios psicológicos, físicos y sociales, donde se enfatiza en la competencia. Es por este motivo, el alumno con creencias fijas se ve menos equipado para superar tales retos (Blackwell et al., 2007).

En ese sentido, en una investigación realizada con alumnos que se encontraban en la transición de la primaria a la secundaria, se reportó que los alumnos con creencia fija presentaban una disminución en sus notas, a comparación de los que tenían una creencia crecencial, quienes obtuvieron una mejoría en sus calificaciones. Los alumnos con creencias fijas tendían a despreciar sus capacidades por las dificultades propias de la educación secundaria, culpándose a sí mismos o a los demás de sus fracasos; en cambio los de mentalidad crecencial, si bien se sentían abrumados por los retos que implicaba la secundaria, su respuesta fue profundizar y hacer lo necesario para incrementar sus aprendizajes, esforzándose más y cambiando de estrategias si la situación lo requería (Dweck, 2017).

En consecuencia, los alumnos con creencias fijas ven la transición de primaria a secundaria como un proceso difícil, como una amenaza, y como una situación en la que pueden ser catalogados de forma negativa. Por ende, el alumno con creencia fija hace uso de sus recursos, no para aprender, sino para proteger su ego, esforzándose menos y evitando el fracaso (Dweck, 2017). 
Por otro lado, Dweck y Elliott (1983) explicaron que la razón por la cual existían alumnos capaces que creían que no lo eran, radicaba en los objetivos de la persona, ya que algunas se orientan hacia sus metas de aprendizaje y otras hacia al resultado. Dweck (2002) demostró también, que elogiar a los niños por su inteligencia, más que por su esfuerzo, socava su motivación y ocasionaba que los niños mientan para obtener elogios. Por consiguiente, Dweck (2007) se preguntó sobre aquello que haría que las personas se centren en determinado objetivo, lo cual le llevó a la conclusión de que las personas que desean demostrar resultados, creerían que su inteligencia es estática, mientras que quienes desean mejorar sus aprendizajes tenían una creencia de la inteligencia como una habilidad dinámica y maleable.

Además, de los estudios de Dweck, se han llevado a cabo varias investigaciones sobre la teoría implícita de la inteligencia en diversos países del mundo. Por ejemplo, Tarbetsky et al. (2016) realizaron una investigación con 87 estudiantes australianos indígenas y 87 estudiantes australianos no indígenas, concluyendo que las creencias implícitas y las habilidades son un factor motivacional que predice el logro académico, siendo los estudiantes indígenas quienes presentaron bajos niveles en rendimiento académico, acompañado del predominio de creencias fijas. Por otro lado, quienes manifestaron mayores creencias incrementales obtuvieron un buen resultado académico, siendo las estudiantes mujeres quienes mostraron un mayor rendimiento general. Estos resultados se contraponen a los que obtuvo Gayón (2010), quien no encontró diferencias significativas entre niños y niñas, como lo indicaron los estudios de Dweck y Leggett (1988), y Leggett (1986), quienes encontraron además, que los escolares de sexo femenino mostraban mayor tendencia por la creencia de tipo fija.

En otro estudio, Galvis et al. (2010) evaluaron a 107 escolares que cursaban los grados quinto, sexto y séptimo de un colegio en Colombia, reportando que hubo predominio de la creencia de tipo de crecimiento mediada por el esfuerzo y el aprendizaje. Mientras que, en una investigación de Lammel y Guillén (2011) se encontró que los niños mexicanos evidenciaron una creencia predominante sobre la inteligencia fija, mientras que los niños franceses tenían puntajes más altos en creencias de la inteligencia incremental. Estos resultados se explicaron, debido a que los niños franceses perciben la inteligencia como algo individual y cognitivo, mientras que los niños mexicanos tienen una conceptualización dual que se conforma por el aspecto social y cognitivo.

A su vez, Wang y Fei-YinNg (2012) realizaron dos estudios cada uno con una duración de seis meses en población china. El primer estudio con 581 estudiantes (231 varones y 350 mujeres) con un promedio de edad de 16 años y en el segundo estudio participaron 361 de los estudiantes de décimo grado (124 niños, 237 niñas), que fueron evaluados con el cuestionario de Dweck (2000). Los estudiantes chinos, tendieron a ver a la 
inteligencia como más inmutable que el rendimiento escolar, y resaltaron la importancia de un enfoque positivo en el trabajo escolar.

\section{Rendimiento académico}

Según Figueroa (2004), el rendimiento académico es el producto de la comprensión del contenido de los programas de estudio, siendo reflejado en calificaciones dentro de una escala convencional (Noack, 2018). Por otro lado, la UNESCO (2015) refiere que el logro del aprendizaje es un componente indispensable que evidencia la calidad educativa. Mientras que para Woolfolk (2010) el rendimiento académico aporta información sobre el aprendizajey de las formas en que integran sus conocimientos, desde niveles de menor a mayor complejidad.

Asimismo, en cuanto al sistema de evaluación escolar peruano, en el nivel de Educación Primaria se hace uso de una escala literal y descriptiva, son los siguientes niveles: AD o "Logro destacado" refiere que el alumno manifiesta los aprendizajes previstos y a su vez, ha superado el nivel esperado en función a la competencia. A o "Logro esperado" se otorga en la medida en que los alumnos consiguen demostrar el nivel esperado de forma provechosa en un tiempo determinado. Asimismo, se obtendrá B o "En proceso" cuando el estudiante está en trayecto a adquirir el aprendizaje, y por ello, requerirá de apoyo en un tiempo programado que le permita conseguirlo. Co "En inicio" se da a aquellos estudiantes que denotan un progreso mínimo en cuanto al aprendizaje de los contenidos, evidenciando inconvenientes para lograrlo, de tal manera que necesitarán de un apoyo por un tiempo más prolongado (Ministerio de Educación del Perú, 2017).

En cuanto a la Educación Secundaria, el Ministerio de Educación de Perú aplica de forma escalonada una calificación académica cualitativa. En efecto, AD significa un logro muy satisfactorio (LMS), A logro satisfactorio (LS), B logro básico (LB) y C logro inicial (I) (Correo, 2019).

En nuestro país, como en otros de Latinoamérica, el rendimiento académico, está fuertemente asociado con la condición socioeconómica de los estudiantes. Así, los factores sociales y económicos contribuyen a explicar el pensamiento de la sociedad y su evolución. Por tanto, es de suma importancia tomarlos en cuenta en las investigaciones educacionales (Dongo, 2009), ya que la carencia de dinero en algunos grupos sociales, tienen por consecuencia crecientes problemas de educación (Rodríguez, 2015).

Una explicación a estas deficiencias educativas mediadas por la condición socioeconómica, tiene que ver con el involucramiento de la familia en el rendimiento de los hijos. Lastre, López y Alcázar (2018) realizaron una investigación que reveló la asociación entre variables de acompañamiento de la familia y rendimiento académico en estudiantes de $3^{\circ}$ de primaria en Colombia; reportando que los alumnos de mayor nivel socioeconómico obtuvieron un rendimiento académico más alto 
debido a que tenían acompañamiento por parte de sus padres.

Asimismo, se llevó a cabo una investigación para indagar la influencia de la estructura familiar en el rendimiento académico en matemática y lectura de los escolares de $6^{\circ}$ de primaria de diferentes países de América Latina, con una muestra de 90 mil niños y sus respectivas familias de 2450 instituciones educativas; concluyéndose que los estudiantes de familias nucleares alcanzan un rendimiento superior en matemática y en lectura en comparación con los alumnos de familias monoparentales. Por otro lado, se halló una correlación positiva entre el nivel educativo de los padres y el rendimiento académico de sus hijos. A su vez, se encontró que a medida que se incrementa el nivel socioeconómico familiar, las diferencias de rendimiento académico entre familias monoparentales y familias nucleares son más notorias, a comparación de sectores pobres que mantienen un rendimiento bajo, independientemente dela estructura familiar (Cervini et al., 2014). Por el contrario, Cruz et al. (2014) reportaron una relación débil entre el desempeño escolary el nivel socio económico.

Tomando en cuenta las variables familiares, se realizó una investigación en España, con 486 escolares de secundaria, entre 12 y 18 años; en el que se reportó que el desempeño académico puede verse influido por las expectativas o percepciones que los padres presentan sobre las posibilidades académicas de sus hijos, siendo la madre la de mayor influencia.
Igualmente se encontró asociación entre el nivel educativo y empleo de los padres con el desempeño académico de sus hijos, obteniendo un efecto positivo en el rendimiento de los hijos, a medida que se incrementan estas variables. Esto se explica, por el hecho, de que las clases sociales medias o acomodadas, cuentan con ingresos familiares suficientes para adquirir los materiales de estudio requeridos (Fajardo et al., 2017). En general, como dice Olmeda (2016), el rendimiento no es una variable independiente del contexto en el que vive el alumno.

En cuanto a la edad, Peña (2015) refiere que los alumnos menores en edad dentro de un mismo grado escolar poseen un desempeño escolar bajo en pruebas estandarizadas. Así mismo, Backhoff, Sánchez, Peón y Andrade (2010) sostienen que los escolares con una edad acorde a su nivel de estudios tienen un mejor rendimiento.

Por otro lado, se debe agregar que, con respecto al sexo, Soto y colaboradores (2015) encontraron que no existe una diferencia significativa entre el rendimiento escolar a pesar que las mujeres tienden a tener calificaciones ligeramente más altas. De manera análoga la Organización para la Cooperación y Desarrollo Económico (OECD, 2013), señala que existe una leve propensión a que las mujeres posean mejores calificaciones y menos disertaciones en el colegio. 


\section{Método}

\section{Diseño}

El diseño de este estudio fue no experimental, transversal y correlacional puesto que no se manipularon variables independientes para generar efectos en variables dependientes, y sólo se observó la asociación entre las variables de estudio en un momento dado (León \& Montero, 2007).

\section{Participantes}

La muestra estuvo conformada por 434 alumnos de quinto y sexto grado de educación primaria y primero de secundaria. La selección de la muestra se hizo por conveniencia. Se eligió a colegios particulares y nacionales, diurnos y mixtos La población a la que se dirigió la presente investigación abarcó niños con un desarrollo típico es decir no tienen algún tipo de dificultad en su desarrollo.

\section{Instrumentos}

Para evaluar las creencias implícitas de la inteligencia se utilizó The Implicit Theory of Intelligence Scale (ITIS) cuyos autores son Abd-El-Fattah y Yates (2006), y está basado en el modelo de Dweck (1999, 2002). La versión inglesa fue validada a la población de Colombia, y fue adaptada y traducida por expertos (Muñiz \& Hambleton, 1996; Flores et al., 2006). La versión que se utilizó en este trabajo constó de 14 ítems, siete de los cuales evalúan creencias fijas y los siete restantes creencias de crecimiento. Esta versión fue validada para estudiantes escolares por Gayón (2010), con un índice de confiabilidad de .90 con el método test-retest.

Para evaluar el rendimientoacadémico delos escolares se dispuso con un formato en el cual se registraron las calificaciones del primer trimestre, en donde figuran el promedio de las calificaciones correspondientes para los cursos de lenguajey matemática; obteniéndose como medida del desempeño escolar (Stromso \& Braten, 2004).

Para el registro del nivel socioeconómico se aplicó una ficha sociodemográfica que elaborada a partir de los datos obtenidos por la APEIM, que constó de cinco niveles, A, B, C, D y E; cada una con una descripción sobre qué se requiere para cada nivel, que permitió obtener información en el aspecto económico sobre el hogar del estudiante. Existen investigaciones en nuestro país que hicieron uso de las variables propuestas por APEIM vara evaluar el nivel socioeconómico que han dado cuenta de su validez y confiabilidad (Hoyos, 2016; Vera \& Vera, 2013; Zegarra, 2013).

\section{Procedimiento}

En primer lugar se coordinó con las autoridades de las instituciones educativas los permisos correspondientes para la ejecución de la investigación. Seguidamente, se procedió a la aplicación de la prueba dentro del horario escolar de los estudiantes. Vale precisar que se pasó a entregar los consentimientos a los padres de familia, esperando una semana para el regreso de éste y poder pasar al asentimiento por parte 
de los alumnos quienes también tenían la libertad de negarse a participar de la investigación. Asimismo junto con el consentimiento de los padres de familia se les entregó dos preguntas referentes a datos socioeconómicos, y las preguntas restantes se recabaron de los datos registrados en la institución educativa. La aplicación de la prueba y la ficha sociodemográfica se dio grupalmente. Finalmente se revisaron los registros de notas de los estudiantes para identificar su desempeño escolar, lo cual permitió diferenciar a los estudiantes de bajo rendimiento escolar.

\section{Criterios para el análisis de datos}

La información recolectada en las encuestas fue ingresada a una matriz de datos en el software Excel. Posteriormente, se procedió a realizar el análisis exploratorio, descriptivo e inferencial de los datos en el software $\mathrm{R}$ v3.5.1. Para comenzar, se estimaron las medidas de tendencia central en las variables cuantitativas y frecuencias porcentuales en las variables cualitativas. De este modo se pudo determinar que ambas dimensiones de las teorías implícitas tienen distribuciones aproximadas a la normalidad (Teorías fijas: $\mathrm{W}=0.95, \mathrm{p}=.45$; Teorías incrementales: $\mathrm{W}=0.93, \mathrm{p}=.20$ ). Al analizar las frecuencias del desempeño académico en los cursos de comunicación y matemática se observaron frecuencias menores a 5 en las notas $\mathrm{AD}$ y $\mathrm{C}$, lo cual genera dificultades en la estimación de modelos estadísticos. Es por este motivo que se recodificó dicha variable con dos categorías: Alto rendimiento (que comprende las notas AD y A) y Bajo rendimiento (que comprende las notas B y C). Del mismo modo, al analizar el nivel socioeconómico de los estudiantes se observaron frecuencias bajas en los estratos A y E, por lo cual se recodificó esta variable, para lo cual se juntaron los estratos A con B, y D con $\mathrm{E}$, para poder tener estimaciones más adecuadas. Además, las comparaciones de promedios se analizaron utilizando $t$ de Student cuando se compararon dos grupos, y ANOVA cuando se compararon más de dos grupos. Para realizar los contrastes específicos de las pruebas de ANOVA se utilizó la prueba post-hoc con ajuste de Bonferroni. Del mismo modo cuando se analizaron las diferencias entre dos variables categóricas se utilizó la prueba de Chi-Cuadrado.

\section{Resultados}

En la Tabla 1 se encuentran los estadísticos descriptivos de los tipos de creencias acerca de la inteligencia reportadas por los estudiantes de la muestra de la presente investigación. Se pudo observar que los participantes reportaron un puntaje estadísticamente significativo mayor en las creencias fijas $(M=2.78)$ que en las creencias incrementales $(M=1.81$, $t=33.88, \mathrm{gl}=433, \mathrm{p}<.001$ ) 
Tabla 1. Valores descriptivos de los tipos de creencias acerca de la inteligencia de los estudiantes

\begin{tabular}{|lcccc|}
\hline \multicolumn{1}{|c}{ Variable } & M & DE & Min & Max \\
\hline Creencias Fijas & 2.78 & 0.41 & 1.29 & 4.00 \\
Creencias Incrementales & 1.81 & 0.40 & 1.00 & 3.14 \\
\hline
\end{tabular}

En la Tabla 2 se pueden observar las diferencias entre los promedios de los tipos de las creencias acerca de la inteligencia entrecada uno de los niveles académicos que fueron encuestados. En ambos tipos, creencias fijas eincrementales, noseobservaron diferencias estadísticamente significativas $\left(F_{(2,431)}=0.97\right.$, $\mathrm{p}=.38 ; F_{(2,431)}=1.50, \mathrm{p}=.22$, respectivamente), por lo que se considera que los promedios son iguales para los tres niveles.

Tabla 2. Comparación de tipos de las creencias acerca de la inteligencia por nivel académico

\begin{tabular}{|c|c|c|c|c|c|c|c|c|}
\hline & \multicolumn{2}{|c|}{$\begin{array}{c}5^{\text {to }} \\
\text { Primaria }\end{array}$} & \multicolumn{2}{|c|}{$\begin{array}{c}\text { 6to } \\
\text { Primaria }\end{array}$} & \multicolumn{2}{|c|}{$\begin{array}{c}\text { 1ro } \\
\text { Secundaria }\end{array}$} & \multirow[t]{2}{*}{$F(2,431)$} & \multirow[t]{2}{*}{$\mathbf{p}$} \\
\hline & $\mathbf{M}$ & DE & $\mathbf{M}$ & DE & $\mathbf{M}$ & DE & & \\
\hline C. Fijas & 1.77 & 0.38 & 1.83 & 0.45 & 1.83 & 0.38 & 0.97 & .38 \\
\hline C. Incrementales & 2.73 & 0.43 & 2.82 & 0.36 & 2.79 & 0.42 & 1.50 & .22 \\
\hline
\end{tabular}

En la Tabla z es posible observar la comparación del rendimientoacadémico en comunicacióny matemática por nivel académico. Se observaron diferencias significativas entrelos participantes, donde los estudiantes de 5 to y 6toen su mayoría tienen un rendimientoalto; mientras que, los estudiantes de primero de secundaria tienen un rendimiento bajo $\left(X_{(2)}^{2}=\right.$ 40.47, p<.001). En estesentidolosestudiantes de secundaria parecen tener mayores dificultades con el rendimiento en este curso. Al igual que en el curso de comunicación, seobservaron diferencias significativas entre los distintos niveles académicos en el curso de matemática. Los estudiantes de 5to y 6to tienen mejor rendimiento académico que los estudiantes 1ro de secundaria $\left(X_{(2)}^{2}=\right.$ $9 \cdot 54, \mathrm{p}=.008)$. 
Tabla 3. Comparación del rendimiento académico en comunicación y matemática por nivel académico

\begin{tabular}{|c|c|c|c|c|c|c|}
\hline & \multicolumn{2}{|c|}{ Alto } & \multicolumn{2}{|c|}{ Bajo } & \multirow{2}{*}{$\mathbf{x} 2(2)$} & \multirow{2}{*}{$\mathbf{p}$} \\
\hline & $\mathbf{N}$ & $\%$ & $\mathbf{N}$ & $\%$ & & \\
\hline \multicolumn{7}{|c|}{ Comunicación } \\
\hline 5to & 71 & 61.2 & 45 & 38.8 & 40.47 & .001 \\
\hline 6 to & 92 & $79 \cdot 3$ & 24 & 20.7 & & \\
\hline 1ero & 87 & 43.0 & 115 & $59 \cdot 9$ & & \\
\hline \multicolumn{7}{|c|}{ Matemática } \\
\hline $5^{\text {to }}$ & 59 & 50.9 & 57 & 49.1 & $9 \cdot 54$ & .008 \\
\hline 6to & 65 & 56.0 & 51 & 44.0 & & \\
\hline 1ero & 79 & 39.0 & 123 & 61.0 & & \\
\hline
\end{tabular}

En la Figura 1 se puede observar la relación entre los tipos de creencias acerca de la inteligencia y el rendimiento académico en comunicación y matemática. Con respecto a las creencias fijas y el curso de comunicación se observaron diferencias significativas de acuerdo con la prueba t de Student $\left(t_{(431)}=\mathbf{2 . 9 1}, \mathrm{p}=.003\right)$. Específicamente los estudiantes con alto desempeño en comunicación tienen un promedio significativamente más alto que el de los estudiantes con un bajo desempeño. Por el contrario, en cuanto a las creencias incrementales se observaron diferencias significativas $\left(t_{(431)}=\mathbf{- 2 . 3 9}, \mathrm{p}=\right.$ .02). En este caso los estudiantes con un bajo desempeño en comunicación, son los que tienen un promedio más alto en este tipo de creencia por sobre los estudiantes con un alto desempeño. Estos dos resultados si bien apuntan a la existencia de una relación entre las creencias acerca de la inteligencia y el rendimiento académico, van en contra de lo propuesto en la hipótesis de la presente investigación. 

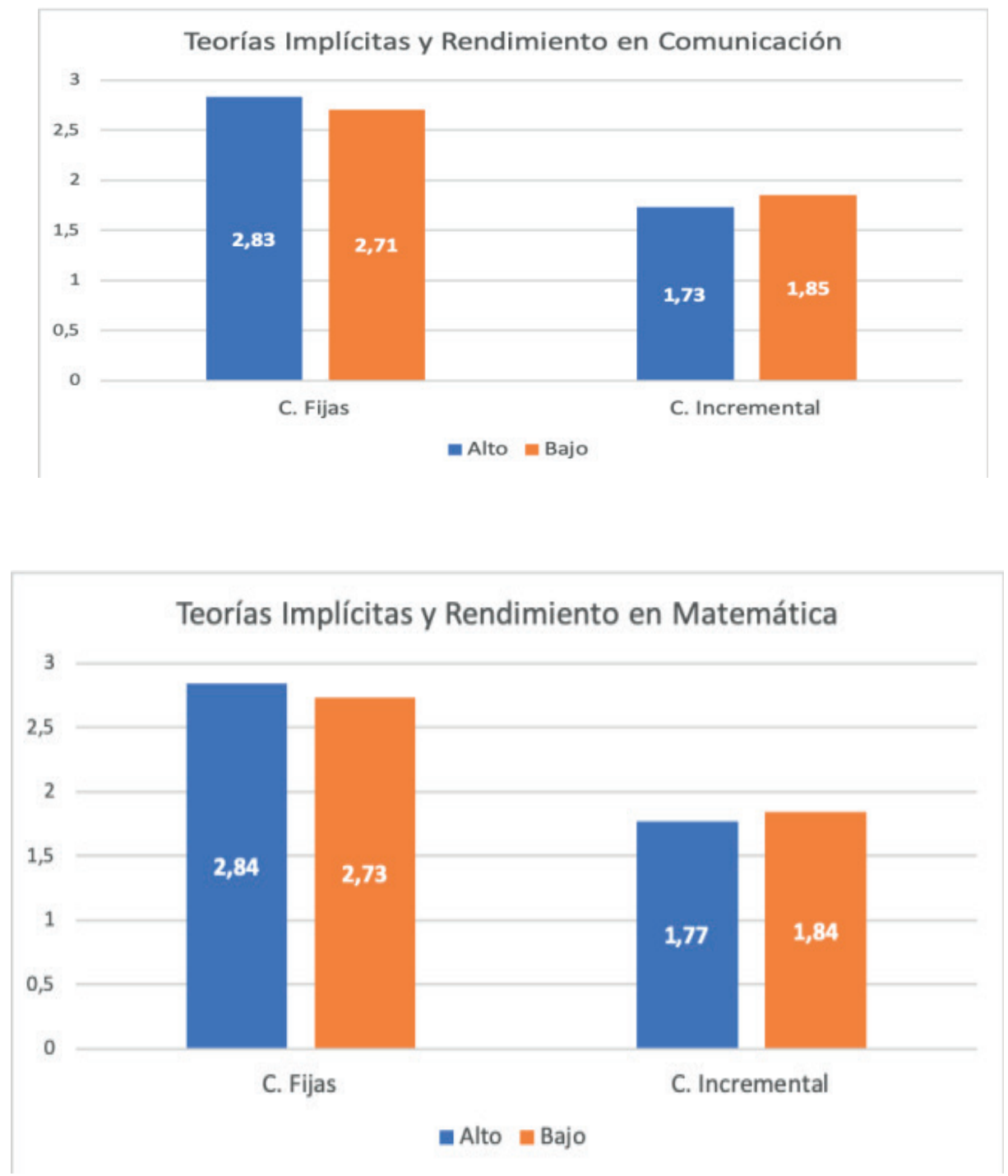

Figura 1. Asociación entre los tipos de creencias acerca de la inteligencia y rendimiento académico en comunicación y matemática

Enel casodel cursodematemáticaseencuentraun patrón similaral observadoen el rendimiento en comunicación. Los estudiantes con alto rendimiento presentan una media significativamente más alta en creencias fijas que los estudiantes con un desempeño bajo $\left(\mathrm{t}_{(431)}=2.84, \mathrm{p}=.005\right)$. Además, los estudiantes con un bajo desempeño en matemática presentan una media significativamente más alta en creencias incrementales que los 


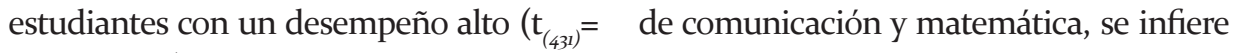
$-2.00, \mathrm{p}=.05)$. Estos resultados nuevamente queal menos en una muestra arequipeña las van en contra de lo que era esperado de creenciasfijasseasocianaunaltodesempeño acuerdocon la revisión de literatura realizada. y las creencias incrementales seasocian a un Yalobservarseel mismo patrón en loscursos desempeño bajo.

Tabla 4.

Comparación de los tipos de creencias acerca de la inteligencia por sexo de los estudiantes

\begin{tabular}{|lcccccc|}
\hline & \multicolumn{2}{c}{ Femenino } & \multicolumn{2}{c}{ Masculino } & \multirow{2}{*}{ (412) } & p \\
& M & DE & M & DE & & .95 \\
C. Fijas & 2.78 & 0.43 & 2.78 & 0.38 & -0.06 & .21 \\
C. Incrementales & 1.84 & 0.42 & 1.79 & 0.38 & 1.26 & .26 \\
\hline
\end{tabular}

En la Tabla 4 se encuentra la compara- significativas por género (Creencias fijas: ción de promedios de los tipos de creen $-t_{(412)}=-0.06, p=.95$; Creencias incremencias acerca de la inteligencia por sexo de tales: $\left.\mathrm{t}_{(412)}=1.26, \mathrm{p}=.21\right)$. Por lo que el los estudiantes. En ninguno de los tipos sexo no es determinante al momento de creencias acerca de la inteligencia se de formar las creencias acerca de la observaron diferencias estadísticamente inteligencia. 
Tabla 5.

Nivel familiar y creencias fijas acerca de la inteligencia

\begin{tabular}{|c|c|c|c|c|}
\hline & $\mathbf{M}$ & DS & $\mathbf{F}$ & $\mathbf{p}$ \\
\hline \multicolumn{5}{|l|}{ Tipo de familia } \\
\hline Nuclear & 1.77 & 0.38 & 0.97 & .38 \\
\hline Extensa & 1.83 & 0.45 & & \\
\hline Monoparental & 1.83 & 0.38 & & \\
\hline \multicolumn{5}{|c|}{ Personas que ayudan al estudiante con las tareas } \\
\hline Padre & 2.81 & 0.43 & 2.92 & .03 \\
\hline Madre & 2.82 & 0.41 & & \\
\hline Otro & 2.81 & 0.35 & & \\
\hline Nadie & 2.68 & 0.43 & & \\
\hline \multicolumn{5}{|c|}{ Frecuencia de ayuda en las tareas } \\
\hline Frecuentemente & 2.77 & 0.44 & 3.75 & .01 \\
\hline$A$ veces & 2.81 & 0.39 & & \\
\hline Rara vez & 2.87 & 0.36 & & \\
\hline Nadie & 2.68 & 0.42 & & \\
\hline \multicolumn{5}{|c|}{ Nivel de instrucción de la persona que ayuda con las tareas } \\
\hline Primaria Incompleta & 2.89 & 0.57 & 2.63 & .02 \\
\hline Primaria Completa & 2.64 & 0.42 & & \\
\hline Secundaria Incompleta & 2.73 & 0.39 & & \\
\hline Secundaria Completa & 2.82 & 0.41 & & \\
\hline Superior Incompleta & 2.90 & 0.41 & & \\
\hline Superior Completa & 2.78 & 0.37 & & \\
\hline Nadie & 2.68 & 0.43 & & \\
\hline \multicolumn{5}{|c|}{ Tipo de ocupación de quien ayuda con las tareas al estudiante } \\
\hline Empresa & 2.80 & 0.31 & 2.64 & .03 \\
\hline Propio & 2.87 & 0.46 & & \\
\hline Eventual & 2.83 & 0.38 & & \\
\hline No trabaja & 2.79 & 0.41 & & \\
\hline Nadie & 2.68 & 0.43 & & \\
\hline \multicolumn{5}{|c|}{ Tiempo de reacción ante los requerimientos de material académico de los estudiantes } \\
\hline Inmediatamente & 2.80 & 0.43 & 0.52 & 67 \\
\hline Tardan & 2.77 & 0.37 & & \\
\hline Falta dinero & 2.81 & 0.59 & & \\
\hline No prestan atención & 2.62 & 0.47 & & \\
\hline \multicolumn{5}{|c|}{ Tiempo de recreación con la familia } \\
\hline Poco & 2.72 & 0.44 & 0.66 & .52 \\
\hline Algo & 2.79 & 0.40 & & \\
\hline Mucho & 2.79 & 0.41 & & \\
\hline
\end{tabular}

En la Tabla 5 es posible observar la de la inteligencia. Con respecto a la tipo comparación las características de nivel de familia no se observaron diferencias y entorno familiar y las creencias acerca significativas $\left(F_{(2,431)}=0.97, \mathrm{p}=.38\right)$, por 
lo que de acuerdo con estos resultados el tipo de familia no tiene implicancias sobre el desarrollo de creencias fijas de la inteligencia. Para la comparación de la persona reportada como quien ayuda al estudiante y las creencias fijas se observan diferencias significativas $\left(F_{(3,430)}=2.92, \mathrm{p}=\right.$ 0.03 ) donde de acuerdo a los contrastes de Bonferroni las diferencias específicas se encuentran entre quienes reciben ayuda de la madre y quienes no reciben ayuda de nadie $(p=.038)$, donde quienes reciben ayuda de la madre tienen un promedio mayor de creencias fijas que quienes no reciben ayuda de nadie.

Para la comparación entre frecuencia de ayuda con las tareas se observaron diferencias significativas en las creencias fijas $\left(F_{(2,431)}=3 \cdot 75, \mathrm{p}=.01\right)$, y de acuerdo con los contrastes post hoc las diferencias específicas se encuentran entre aquellos participantes que reciben ayuda rara vez contra los que no reciben ayuda de ningún tutor con creencias fijas más altas para los primeros $(p=.01)$. Del mismo modo para el nivel de instrucción de quien ayuda con las tareas y las creencias fijas se observan diferencias significativas $\left(F_{(5,428)}=2.63, \mathrm{p}=\right.$
.02), donde de acuerdo a los contrastes post-hoc las diferencias específicas se encuentran entre aquellos tutores que tienen un nivel de instrucción superior incompleta frente a los que no reciben ayuda de nadie ( $\mathrm{p}=.007$ ) siendo los primeros quienes muestran un promedio superior al segundo.

Con respecto a las creencias fijas de la inteligencia y el tipo de ocupación de quien ayuda en las tareas, se observan diferencias estadísticamente significativas $\left(F_{(4,429)}=2.64, \mathrm{p}=.03\right)$, donde de acuerdo a los contrastes post hoc, las diferencias específicas se encuentran entre aquellos que tienen un negocio propio y aquellos que no reciben ayuda de ningún familiar o tutor $(p=.04)$, siendo los primeros quienes tienen un promedio de creencias fijas más alto que los segundos. Para la relación entre el tiempo de reacción de frente a las necesidades académicas del estudiantey las creencias fijas de la inteligencia no se observaron diferencias $\left(F_{(3,430)}=.52, \mathrm{p}=\right.$ $.67)$. Del mismo modo para la relación entre creencias fijas y tiempo de recreación con la familia, no se observaron diferencias significativas $\left(F_{(2,431)}=0.66, \mathrm{p}=.52\right)$. 
Tabla 6.

Nivel familiar y creencias incrementales acerca de la inteligencia

\begin{tabular}{|c|c|c|c|c|}
\hline & M & DS & $\mathbf{F}$ & p \\
\hline \multicolumn{5}{|l|}{ Tipo de familia } \\
\hline Nuclear & 2.73 & 0.43 & 1.50 & .22 \\
\hline Extensa & 2.82 & 0.36 & & \\
\hline Monoparental & 2.79 & 0.42 & & \\
\hline \multicolumn{5}{|c|}{ Personas que ayudan al estudiante con las tareas } \\
\hline Padre & 1.80 & 0.41 & 0.32 & .81 \\
\hline Madre & 1.82 & 0.40 & & \\
\hline Otro & 1.78 & 0.42 & & \\
\hline Nadie & 1.83 & 0.35 & & \\
\hline \multicolumn{5}{|c|}{ Frecuencia de ayuda en las tareas } \\
\hline Frecuentemente & 1.82 & 0.40 & 0.13 & .93 \\
\hline A veces & 1.80 & 0.41 & & \\
\hline Rara vez & 1.80 & 0.42 & & \\
\hline Nadie & 1.83 & 0.36 & & \\
\hline \multicolumn{5}{|c|}{ Nivel de instrucción de la persona que ayuda con las tareas } \\
\hline Primaria Incompleta & 1.89 & 0.52 & 0.40 & .88 \\
\hline Primaria Completa & 1.67 & 0.32 & & \\
\hline Secundaria Incompleta & 1.78 & 0.43 & & \\
\hline Secundaria Completa & 1.79 & 0.34 & & \\
\hline Superior Incompleta & 1.84 & 0.43 & & \\
\hline Superior Completa & 1.81 & 0.43 & & \\
\hline Nadie & 1.83 & 0.36 & & \\
\hline \multicolumn{5}{|c|}{ Tipo de ocupación de quien ayuda con las tareas al estudiante } \\
\hline Empresa & 1.75 & 0.38 & 1.29 & .27 \\
\hline Propio & 1.85 & 0.42 & & \\
\hline Eventual & 1.85 & 0.39 & & \\
\hline No trabaja & 1.84 & 0.45 & & \\
\hline Nadie & 1.83 & 0.35 & & \\
\hline \multicolumn{5}{|c|}{ Tiempo de reacción ante los requerimientos de material académico de los estudiantes } \\
\hline Inmediatamente & 1.79 & 0.40 & 2.02 & .11 \\
\hline Tardan & 1.83 & 0.38 & & \\
\hline Falta dinero & 2.07 & 0.62 & & \\
\hline No prestan atención & 1.64 & 0.35 & & \\
\hline \multicolumn{5}{|c|}{ Tiempo de recreación con la familia } \\
\hline Poco & 1.93 & 0.49 & 3.75 & .02 \\
\hline Algo & 1.83 & 0.38 & & \\
\hline Mucho & 1.77 & 0.39 & & \\
\hline
\end{tabular}

En la Tabla 6 es posible observar la de la inteligencia. Con respecto a la tipo comparación las características de nivel de familia no se observaron diferencias familiar y las creencias incrementales significativas $\left(F_{(2,431)}=1.50, \mathrm{p}=.22\right)$, por lo 
que de acuerdo con estos resultados el tipo de familia no tiene implicancias sobre el desarrollo de creencias incrementales de la inteligencia. Para la comparación de la persona reportada como quien ayuda al estudiante y las creencias incrementales tampoco se observaron diferencias significativas $\left(F_{(2,431)}=0.32, \mathrm{p}=.81\right)$. Del mismo modo para la comparación entre frecuencia de ayuda con las tareas no se observaron diferencias significativas en las creencias incrementales $\left(F_{(2,431)}=.13, \mathrm{p}=.93\right.$. Para el nivel de instrucción de quien ayuda con las tareasy las creencias incrementales tampoco se observan diferencias significativas $\left(F_{(5,428)}=0.40, \mathrm{p}=.88\right)$. Con respecto a las creencias incrementales y el tipo de ocupación de quien ayuda en las tareas, no se observan diferencias estadísticamente significativas $\left(F_{(4,429)}=1.29, \mathrm{p}=.27\right)$. Para la relación entre el tiempo de reacción frente a las necesidades académicas del estudiante y las creencias incrementales de la inteligencia no se observaron diferencias $\left(F_{(3,430)}=2.02, \mathrm{p}=.11\right)$. Sin embargo, se observan diferencias estadísticamente significativas en la dimensión de creencias incrementales $\left(F_{(2,431)}=3.75, \mathrm{p}=.02\right)$, de acuerdo con los contrastes post-hoc las diferencias específicas se encuentran entre los grupos de mucho tiempo con la familia frente a poco tiempo con la familia $(\mathrm{p}=0.03)$; siendo los últimos quienes presentan un promedio superior en este tipo de creencias. Por lo cual de acuerdo con estos resultados el tiempo que pasen los hijos con la familia impacta sobre el desarrollo de creencias incrementales de la inteligencia.

Tabla 7.

Comparación del NSE de los estudiantes y los tipos de creencias acerca de la inteligencia

\begin{tabular}{|lccccc|}
\hline & \multicolumn{2}{c}{ C. Fijas } & \multicolumn{2}{c|}{ C. Incrementales } \\
& $\boldsymbol{M}$ & $\boldsymbol{D} \boldsymbol{E}$ & $\boldsymbol{M}$ & $\boldsymbol{D} \boldsymbol{E}$ \\
\hline A / B & 2.76 & 0.44 & 1.76 & 0.38 \\
C & 2.81 & 0.43 & 1.82 & 0.40 \\
D / E & 2.68 & 0.41 & 1.80 & & 0.41 \\
$F_{(4,429)}$ & & 0.49 & & 0.41 & \\
$P$ & & & & \multicolumn{2}{c}{.80} \\
\hline
\end{tabular}

En la Tabla 7 es posible ver la comparación de promedios de los dos tipos de creencias acerca de la inteligencia con el nivel socioeconómico de los estudiantes. En ninguno de los dos tipos de creencias se pudieron observar diferencias significativas (Creencias fijas: $F_{(4,429)}=1.49$, $\mathrm{p}=.21$; Creencias incrementales: $F_{(4,429)}=$ $0.41, \mathrm{p}=.80$ ), esto indica que independientemente del estrato económico del que provenga la persona, no afecta al tipo de creencias de la inteligencia. 
Tabla 8.

Comparación de los tipos de creencias acerca de la inteligencia y tipo de colegio

\begin{tabular}{|lcccccc|}
\hline & \multicolumn{2}{c}{ Estatal } & \multicolumn{2}{c}{ Particular } & \multirow{2}{*}{ T(431) } & P \\
\hline T. Fijas & M & DE & M & DE & & .03 \\
T. Incrementales & 2.73 & 0.40 & 2.82 & 0.41 & -2.21 & .05 \\
\hline
\end{tabular}

En la Tabla 8 se observa la comparación entre los tipos de creencias acerca de la inteligencia y tipo de colegio con el tipo de colegio. Para las creencias fijas se pudo observar que los estudiantes de colegios particulares tienen un promedio significativamente mayor que los estudiantes de colegios estatales $\left(t_{(431)}=-2.21, \mathrm{p}=.03\right)$. Por el contrario, para las creencias incrementales se observó un promedio significativamente mayor para los estudiantes de los colegios estatales por sobre los estudiantes del colegio particular $\left(t_{(431)}=\right.$ 1.99, $\mathrm{p}=.05)$.

Tabla 9.

Comparación del rendimiento académico en comunicación y matemática por sexo de los estudiantes

\begin{tabular}{|c|c|c|c|c|c|c|}
\hline & \multicolumn{2}{|c|}{ Alto } & \multicolumn{2}{|c|}{ Bajo } & \multirow{2}{*}{$\mathbf{X}^{2}(\mathbf{1})$} & \multirow[b]{2}{*}{$\mathbf{p}$} \\
\hline & $\mathbf{N}$ & $\%$ & $\mathbf{N}$ & $\%$ & & \\
\hline \multicolumn{7}{|c|}{ Comunicación } \\
\hline Femenino & 132 & 64.4 & 73 & 35.6 & 6.81 & .009 \\
\hline Masculino & 118 & 51.5 & 111 & 48.5 & & \\
\hline \multicolumn{7}{|l|}{ Matemática } \\
\hline Femenino & 103 & 50.2 & 102 & 49.8 & 1.62 & .20 \\
\hline Masculino & 100 & $43 \cdot 7$ & 129 & 56.3 & & \\
\hline
\end{tabular}

En la Tabla 9 se puede observar las diferencias de sexo en el rendimiento académico del curso de comunicación y matemática. Se observaron diferencias significativas $\left(X_{(1)}^{2}=6.81, \mathrm{p}=.009\right)$ donde las mujeres obtuvieron un mejor rendimiento académico que el de los hombres en el curso de comunicación. En lo que respecta al curso de matemática no se pudieron observar diferencias significativas $\left(X_{(1)}^{2}=1.62, \mathrm{p}=.20\right)$, por lo que se considera que las notas de hombres y mujeres en matemática son estadísticamente similares. 
Tabla 10.

Rendimiento académico en comunicación y el nivel familiar

\begin{tabular}{|c|c|c|c|c|c|c|}
\hline & \multicolumn{2}{|c|}{ Alto } & \multicolumn{2}{|c|}{ Bajo } & \multirow[b]{2}{*}{$\mathbf{X}^{2}$} & \multirow[b]{2}{*}{$\mathbf{p}$} \\
\hline & $\mathbf{N}$ & $\%$ & $\mathbf{N}$ & $\%$ & & \\
\hline \multicolumn{7}{|l|}{ Tipo de familia } \\
\hline Nuclear & 110 & $54 \cdot 7$ & 91 & $45 \cdot 3$ & 7.09 & .03 \\
\hline Extensa & 100 & 65.8 & 52 & 34.2 & & \\
\hline Monoparental & 40 & $49 \cdot 4$ & 41 & 50.6 & & \\
\hline \multicolumn{7}{|c|}{ Personas que ayudan al estudiante con las tareas } \\
\hline Padre & 38 & $59 \cdot 4$ & 26 & 40.6 & 8.31 & .04 \\
\hline Madre & 105 & 61.4 & 66 & 38.6 & & \\
\hline Otro & 58 & 63.0 & 34 & $37 \cdot 0$ & & \\
\hline Nadie & 49 & 45.8 & 58 & 54.2 & & \\
\hline \multicolumn{7}{|c|}{ Frecuencia de ayuda en las tareas } \\
\hline Frecuentemente & 50 & 55.6 & 40 & $44 \cdot 4$ & $9 \cdot 99$ & .02 \\
\hline A veces & 105 & $64 \cdot 4$ & 58 & $35 \cdot 6$ & & \\
\hline Rara vez & 46 & $64 \cdot 4$ & 28 & $35 \cdot 6$ & & \\
\hline Nadie & 49 & 45.8 & 58 & 54.2 & & \\
\hline \multicolumn{7}{|c|}{ Nivel de instrucción de la persona que ayuda con las tareas } \\
\hline Primaria Incompleta & 3 & 60.0 & 2 & 40.0 & 10.01 & .12 \\
\hline Primaria Completa & 7 & 70.0 & 3 & 30.0 & & \\
\hline Secundaria Incompleta & 18 & 60.0 & 12 & 40.0 & & \\
\hline Secundaria Completa & 42 & 55.2 & 34 & $44 \cdot 7$ & & \\
\hline Superior Incompleta & 49 & $63 \cdot 3$ & 28 & 36.4 & & \\
\hline Superior Completa & 82 & 63.6 & 47 & 36.4 & & \\
\hline Nadie & 49 & 45.8 & 58 & 54.2 & & \\
\hline \multicolumn{7}{|c|}{ Tipo de ocupación de quien ayuda con las tareas al estudiante } \\
\hline Empresa & 82 & 61.2 & 52 & 38.8 & 9.62 & .05 \\
\hline Propio & 35 & $59 \cdot 3$ & 24 & 40.7 & & \\
\hline Eventual & 30 & $57 \cdot 7$ & 22 & 42.3 & & \\
\hline No trabaja & 54 & 66.7 & 27 & $33 \cdot 3$ & & \\
\hline Nadie & 49 & 45.8 & 58 & 54.2 & & \\
\hline \multicolumn{7}{|c|}{ Tiempo de reacción ante los requerimientos de material académico de los estudiantes } \\
\hline Inmediatamente & 149 & 61.3 & 94 & 38.7 & 6.52 & .09 \\
\hline Tardan & 95 & $54 \cdot 3$ & 80 & $45 \cdot 7$ & & \\
\hline Falta dinero & 5 & 50.0 & 5 & 50.0 & & \\
\hline No prestan atención & 1 & 16.7 & 5 & $83 \cdot 3$ & & \\
\hline \multicolumn{7}{|c|}{ Tiempo de recreación con la familia } \\
\hline Poco & 22 & 46.8 & 25 & 53.2 & 2.57 & .27 \\
\hline Algo & 106 & 59.6 & 72 & 40.4 & & \\
\hline Mucho & 122 & 58.4 & 87 & 41.6 & & \\
\hline
\end{tabular}

En la Tabla 1o se observan las frecuencias con el rendimiento académico en el curso y porcentajes del nivel familiar comparado de comunicación. De acuerdo con estos 
resultados se observa una relación significativa entre tipo de familia y rendimiento $\left(X_{(2)}^{2}=7.09, p=.03\right)$, donde las familias extensas son las que tienen un porcentaje mayor de hijos con desempeño académico alto en este curso, mientras que las familias nucleares y monoparentales tienen cantidades cercanas al $50 \%$ en ambos tipos de desempeño. De este modo se establece una relación entre el tipo de familia y el desempeño en este curso. La prueba de Chi-Cuadrado también arrojó resultados estadísticamente significativos para la relación entre desempeñoy personas que ayudan al estudiante con las tareas $\left(X_{(3)}^{2}=\right.$ 8.31, $\mathrm{p}=.04)$. Específicamente se observa que aquellos estudiantes que reciben algún apoyo sean de padre, madre u otro tutor tienen un desempeño académico mejor que aquellos que no reciben ayuda de nadie. Esto indica que la atención que brindan los padres a las tareas que tienen los hijos es importante para el desempeño al menos en el curso de comunicación. Se observa una relación significativa entre el rendimiento y frecuencia de ayuda en las tareas $\left(X_{(3)}^{2}=9.99, \mathrm{p}=.02\right)$, donde las familias que ayudan a veces y rara vez a los estudiantes muestran un desempeño académico más alto que aquellos que reciben ayuda frecuentemente o que no reciben ayuda en lo absoluto. Esto indicaría que la cantidad de veces que el estudiante afecta también sobre el desempeño en este curso, siendo necesario moderar la cantidad de veces que el estudiante debe ser ayudado. Para la relación entre el nivel de instrucción y rendimiento no se observaron diferencias significativas $\left(X_{(6)}^{2}=10.01, \mathrm{p}=.12\right)$, indicando que, si bien la ayuda es importante para el desempeño en este curso, el nivel de instrucción de los padres no es un factor determinante. La prueba de Chi-Cuadrado arrojó resultados significativos para la relación entre rendimiento y el tipo de ocupación del quien ayuda con las tareas $\left(X_{(2)}^{2}=9.62, p=.05\right)$. Específicamente se puede observar que aquellos padres que no trabajan logran que la mayoría de sus hijos tengan un desempeño alto, seguido por aquellos padres que son empleados en una empresa. Indicando que probablemente el tiempo que tienen disponible los padres para poder ayudar a sus hijos tiene implicancias sobre el desempeño al menos en el curso de comunicación. La relación entre la respuesta ante necesidad de material académico y el desempeño no resultó significativa $\left(X_{(3)}^{2}=6.52, \mathrm{p}=\right.$ .09 ). No se pudieron observar diferencias significativas en la relación entre tiempo de recreación con la familia y rendimiento $\left(X_{(2)}^{2}=2.57, \mathrm{p}=.27\right)$, por lo que estas dos variables no estarían vinculadas o no tendrían implicancia una sobre la otra. 


\section{Tabla 11.}

\section{Rendimiento académico matemática y nivel familiar}

\begin{tabular}{|c|c|c|c|c|c|c|}
\hline & \multicolumn{2}{|c|}{ Alto } & \multicolumn{2}{|c|}{ Bajo } & \multirow[b]{2}{*}{$\mathbf{X}^{2}$} & \multirow[b]{2}{*}{$\mathbf{p}$} \\
\hline & $\mathbf{N}$ & $\%$ & $\mathbf{N}$ & $\%$ & & \\
\hline \multicolumn{7}{|l|}{ Tipo de familia } \\
\hline Nuclear & 100 & 49.8 & 101 & 50.2 & 17.99 & .001 \\
\hline Extensa & 82 & 53.9 & 70 & 46.1 & & \\
\hline Monoparental & 21 & $25 \cdot 9$ & 60 & 74.1 & & \\
\hline \multicolumn{7}{|c|}{ Personas que ayudan al estudiante con las tareas } \\
\hline Padre & 31 & 48.4 & 33 & 51.6 & 4.12 & .24 \\
\hline Madre & 85 & $49 \cdot 7$ & 86 & 50.3 & & \\
\hline Otro & 46 & 50.0 & 46 & 50.0 & & \\
\hline Nadie & 41 & 38.3 & 66 & 61.7 & & \\
\hline \multicolumn{7}{|c|}{ Frecuencia de ayuda en las tareas } \\
\hline Frecuentemente & 44 & 48.9 & 46 & 51.1 & 4.10 & .25 \\
\hline$A$ veces & 81 & $49 \cdot 7$ & 82 & 50.3 & & \\
\hline Rara vez & 37 & 50.0 & 37 & 50.0 & & \\
\hline Nadie & 41 & 38.3 & 66 & 61.7 & & \\
\hline \multicolumn{7}{|c|}{ Nivel de instrucción de la persona que ayuda con las tareas } \\
\hline Primaria Incompleta & 3 & 60.0 & 2 & 40.0 & 12.17 & .06 \\
\hline Primaria Completa & 4 & 40.0 & 6 & 60.0 & & \\
\hline Secundaria Incompleta & 17 & 56.7 & 13 & $43 \cdot 3$ & & \\
\hline Secundaria Completa & 31 & 40.8 & 45 & 50.2 & & \\
\hline Superior Incompleta & 47 & 61.0 & 30 & 39.0 & & \\
\hline Superior Completa & 60 & 46.5 & 69 & $53 \cdot 5$ & & \\
\hline Nadie & 41 & 38.3 & 66 & 61.7 & & \\
\hline \multicolumn{7}{|c|}{ Tipo de ocupación de quien ayuda con las tareas al estudiante } \\
\hline Empresa & 58 & $43 \cdot 3$ & 76 & 56.7 & 9.41 & .06 \\
\hline Propio & 30 & 50.8 & 29 & 49.2 & & \\
\hline Eventual & 26 & 50.0 & 26 & 50.0 & & \\
\hline No trabaja & 48 & 59.2 & 33 & 40.7 & & \\
\hline Nadie & 41 & 38.3 & 66 & 61.7 & & \\
\hline \multicolumn{7}{|c|}{ Tiempo de reacción ante los requerimientos de material académico de los estudiantes } \\
\hline Inmediatamente & 121 & 49.8 & 122 & 50.2 & 11.61 & .008 \\
\hline Tardan & 81 & 46.3 & 94 & $53 \cdot 7$ & & \\
\hline Falta dinero & 1 & 10.0 & 9 & 90.0 & & \\
\hline No prestan atención & o & o.o & 6 & 100 & & \\
\hline \multicolumn{7}{|c|}{ Tiempo de recreación con la familia } \\
\hline Poco & 16 & 34.0 & 31 & 66.0 & 3.47 & .17 \\
\hline Algo & 87 & 48.9 & 91 & 51.1 & & \\
\hline Mucho & 100 & 47.8 & 109 & 52.2 & & \\
\hline
\end{tabular}

En la Tabla 11 se puede ver la comparación familia y el rendimiento $\left(X_{(2)}^{2}=17.99\right.$, $\mathrm{p}<$ de nivel familiary rendimiento académico .oo1), donde las familias monoparentales en el curso de matemática. Se observa tienen mayores dificultades con el desemuna relación significativa entre el tipo de peño en este curso, siendo el $71 \%$ de sus 
hijos quienes tienen un desempeño bajo. Mientras que en las familias nucleares y extensas no es posible determinar un patrón ya que en ambos casos son cercanos al 50\% para ambos desempeños. De acuerdo con los resultados no se observa una relación significativa entre estas, el desempeño y persona que ayuda al estudiante con las tareas $\left(X_{(3)}^{2}=4 \cdot 12, \mathrm{p}=.24\right)$. Lo cual indica que la atención y ayuda que los padres puedan brindar a sus hijos en el curso de matemática no es necesariamente determinante sobre el desempeño académico sobre el curso de matemática. Del mismo modo, la prueba de Chi-Cuadrado entregó resultados no significativos para la relación entre frecuencia de ayuda con las tareas y rendimiento en matemática $\left(X_{(3)}^{2}=4.10, \mathrm{p}=.25\right)$, lo cual indica que particularmente para el curso de matemática la cantidad de veces que el estudiante reciba ayuda de sus padres o tutores no afecta significativamente a su desempeño académico. Para el nivel de instrucción y rendimiento no se observó una relación significativa $\left(X^{2}{ }_{(6)}=12.17, \mathrm{p}=.06\right)$, por lo que tampoco habría implicancia del nivel de instrucción del padre o tutor sobre el rendimiento académico en matemática. Para la relación entre tipo de ocupación de quien ayuda con las tareas y rendimiento no se observaron diferencias significativas $\left(X_{(4)}^{2}=9.41, \mathrm{p}=.06\right)$, por lo que para el curso de matemática el tipo de trabajo o tiempo que tengan, los padres o tutores, disponible para poder ayudar a los estudiantes no está relacionado al desempeño académico. La prueba de Chi-Cuadrado arrojó un resultado significativo para la relación entre tiempo de reacción ante los requerimientos de material académico y rendimiento $\left(X_{(2)}^{2}=11.61, \mathrm{p}=.008\right)$. Específicamente se observa que los padres que atienden inmediatamente o tardan un poco tienen un desempeño en matemática significativamente más alto que aquellos que no tienen dinero o no prestan atención. Finalmente, para la relación entre tiempo de recreación con la familia y rendimiento no se observó un resultado estadísticamente significativo $\left(X_{(2)}^{2}=3.47\right.$, $\mathrm{p}=.17$ ). 
Tabla 12.

Comparación del NSE de los estudiantes y el rendimiento académico en comunicación y matemática

\begin{tabular}{|c|c|c|c|c|c|c|}
\hline & \multicolumn{2}{|c|}{ Alto } & \multicolumn{2}{|c|}{ Bajo } & \multirow{2}{*}{$X^{2}(4)$} & \multirow{2}{*}{$\mathbf{p}$} \\
\hline & $\mathbf{N}$ & $\%$ & $\mathbf{N}$ & $\%$ & & \\
\hline \multicolumn{7}{|c|}{ Comunicación } \\
\hline $\boldsymbol{A} / \boldsymbol{B}$ & 89 & 64.5 & 49 & $35 \cdot 5$ & 4.65 & .32 \\
\hline C & 96 & 56.1 & 75 & 43.9 & & \\
\hline$D / E$ & 65 & 52.0 & $6 o$ & 48.0 & & \\
\hline \multicolumn{7}{|c|}{ Matemática } \\
\hline $\boldsymbol{A} / \boldsymbol{B}$ & 65 & 47.1 & 73 & 52.9 & 1.16 & .88 \\
\hline C & 76 & 44.4 & 95 & 55.6 & & \\
\hline$D / E$ & 62 & 49.6 & 63 & 50.4 & & \\
\hline
\end{tabular}

En la Tabla 12 se observan las frecuencias y porcentajes del nivel socioeconómico comparado con el rendimiento académico de los estudiantes en comunicación y matemática. En el caso de comunicación no se pudo observar una relación significativa $\left(X_{(2)}^{2}=4.65, \mathrm{p}=.32\right)$, indicando que el rendimiento académico en este curso no está relacionado al estrato socioeconómico del que provenga el estudiante. $\mathrm{Al}$ igual que con el curso de comunicación, no se observó una relación significativa entre el rendimiento en matemática y nivel socioeconómico $\left(X_{(4)}^{2}=1.16, \mathrm{p}=.88\right)$.

Tabla 13.

Comparación del rendimiento académico en comunicación y matemáticas y tipo de colegio

\begin{tabular}{|c|c|c|c|c|c|c|}
\hline & \multicolumn{2}{|c|}{ Alto } & \multicolumn{2}{|c|}{ Bajo } & \multirow{2}{*}{$\mathbf{X}^{2}(\mathbf{1})$} & \multirow{2}{*}{$\mathbf{p}$} \\
\hline & $\mathbf{N}$ & $\%$ & $\mathbf{N}$ & $\%$ & & \\
\hline \multicolumn{7}{|c|}{ Comunicación } \\
\hline Estatal & 103 & 49.8 & 104 & 50.2 & $9 \cdot 36$ & .002 \\
\hline Particular & 147 & 64.8 & 80 & 35.2 & & \\
\hline \multicolumn{7}{|l|}{ Matemática } \\
\hline Estatal & 86 & 41.5 & 121 & 58.5 & 3.95 & .05 \\
\hline Particular & 117 & 51.5 & 110 & 48.5 & & \\
\hline
\end{tabular}

En la Tabla 13 se encuentra la comparación del rendimiento académico en comunicación y matemática y el tipo de establecimiento educativo. La prueba de Chi-Cuadrado indicó que existe una relación estadísticamente significativa $\left(X_{(1)}^{2}=\right.$ $9 \cdot 36, \mathrm{p}=.002)$, donde específicamente se observa que los estudiantes de colegios particulares en su mayoría tienen un desempeño alto en el curso de matemática mientras que los estudiantes de colegios estatales se encuentran divididos entre los dos tipos de desempeño. Esto parecería indicar que hay 
diferencias en el tipo de enseñanza o tipo de evaluación que se realiza en estos colegios. Al igual que con el curso de comunicación se observan diferencias significativas en matemática $\left(X_{{ }_{(4}}{ }^{2}=\right.$ 3.95, $\mathrm{p}=.05)$, donde los estudiantes de colegios estatales tienen un desempeño significativamente más bajo que los estudiantes de los colegios particulares. Esto nuevamente indica que las diferencias educativas y de evaluación entre los colegios no solamente se limitan al curso de comunicación, sino también al curso de matemática.

\section{Discusión}

El objetivo general de la presente investigación fue determinar la asociación entre las creencias implícitas de inteligencia y el rendimiento académico de los estudiantes, dado que varios estudios han demostrado la existencia de efectos positivos en función de las teorías implícitas de inteligencia de los estudiantes sobre sus resultados académicos (Dweck, 2007). Sin embargo, los resultados de la presente investigación fueron opuestos, dado que seencontró que los alumnos con una creencia de crecimiento sobre la inteligencia presentan un bajo rendimiento en ambos cursos, mientras que los escolares con creencia fija alcanzaron un mejor desempeño en ambas asignaturas. Cabe la posibilidad de que, al corresponder las notas de los cursos a la primera parte del año escolar, el nivel de exigencia no fue altamente desafiante como para ver si las creencias incrementales y fijas presentan diferencias en su predicción del rendimiento académico (Blackwell et al., 2007).
Así también a través de investigaciones longitudinales llevadas a cabo por Dweck (2017), se determinó que luego del periodo de transición de primaria a secundaria se evidenciaba que los que poseían una creencia de crecimiento manifestaban luego de dos años de seguimiento un mejor rendimiento. Asimismo, Hong et al. (1999) comentaron que la teoría implícita posee diferentes características para los rasgos de los seres humanos por lo que una persona puede poseer un tipo de creencia para la inteligencia y otra para la asignatura de matemática. Por otro lado, es importante señalar que Hong et al. (1999), Athantic (2017) y Dweck (2017), han sugerido de alguna forma, que la razón por la cual se obtiene resultados opuesto a lo predicho por Dweck radicaría en que las creencias están aliadas a constructos o estructuras de conocimiento (atribuciones y metas). Por lo que si bien de acuerdo a lo estipulado, una creencia fija estaría conectada con una atribución negativa y a una orientación de metas al resultado, tendría un impacto negativo en el rendimiento académico: y no se daría el mismo escenario si el individuo también posee una creencia fija acerca de su inteligencia pero la vinculación hacia estas estructuras es débil o que contrariamente le dé mayor importancia al aprendizaje y al esfuerzo como haría una persona con creencia incremental acerca de su inteligencia (Dweck et al.,1995).

Por otro lado, la investigación llevada a cabo por Castella y Byrne (2015) concluyó que el creer que es posible mejorar la inteligencia no es lo mismo que el estudiante crea personalmente que puede 
mejorar su propia inteligencia. Por ende es importante mencionar que las creencias de crecimiento de la inteligencia según Dweck no se refieren solo al esfuerzo sino que precisa de otros aspectos como es el uso de estrategias nuevas y otros recursos disponibles (Dweck, 2017), de manera que el esfuerzo junto con los otros medios creen el progreso del aprendizaje, refutando la idea de que todos tenemos exactamente el mismo potencial en todos los ámbitos o que podemos aprender todo con la misma facilidad (Blackwell et al., 2007). Es así que la posesión de una creencia de crecimiento significa una real comprensión del nivel de desarrollo de las habilidades de forma realista a pesar de los obstáculos o fracasos. Es importante indicar que Dweck (2017) destaca que la creencia fija es ventajosa debido a que la convicción de querer lograr resultados implica mayor autoexigencia aunque su desventaja será no contribuir a arriesgarse a crecer de una forma más integral en diferentes ámbitos nuevos que permitan aumentar la capacidad de resiliencia y estabilice la confianza en las personas a pesar de no obtener los resultados esperados.

Asimismo, Gayón (2010) evidenció que existe una tendencia por la creencia de tipo fija en general, como se ha encontrado en este estudio. Sin embargo esta no se expresa de forma diferenciada según el sexo, tipo de creencia fija y crecencial en los tres niveles 5to, 6to de primaria y ro de secundaria, pues los resultados no fueron significativos. Lo que podría ser explicado a través del estudio de King (2019) quien refiere que el contagio social de las creencias implícitas de la inteligencia entre compañeros de clases cumple un rol determinante, pudiendo hacer a los estudiantes más susceptibles a las influencias de sus pares. Habría que decir también que el factor cultural tiene que ver en gran medida con los resultados, como lo sustentan Lammel y Guillén (2011), quienes hallaron que la percepción sobre la inteligencia, depende de diferentes realidades y las predisposiciones cognitivas del niño, estando en consonancia con lo expuesto por Dweck (200o), para quien la sociedad en general se dirige a insertar la idea de una creencia fija de la inteligencia como inmutable, pudiendo ser internalizada por los niños y por ende tendiendo a dicha creencia. Teniendo en cuenta que en nuestro país, las prácticas pedagógicas y el desenvolvimiento de los escolares muchas veces no se enfocan en los procesos sino en los resultados, pues muchos de ellos solo buscan aprobar los cursos en lugar de aprender los contenidos enseñados (Arias, 2014).

Además, se reafirmó a través de nuestro estudio lo que Dweck (2017) señala en cuanto a las diferencias en las calificaciones entre primaria y secundaria, lo que se refleja en el mejor rendimiento académico en términos generales en los escolares de 5 to y 6 to de primaria. Por ende, los estudiantes mayores obtuvieron un desempeño más bajo que los estudiantes más jóvenes, debido a que se reduce la intervención de los padres en la educación de los mayores (Sánchez et al., 2010) como ha sido probado en la presente investigación. En tal sentido, algunos desafíos en el nivel secundario se relacionan con la variedad 
de maestros y las nuevas metodologías de enseñanza que se emplean en este nivel escolar (Blackwell et al., 2007). Del mismo modo se halló que hay un mayor desempeño académico en el curso de comunicación en la mayoría de estudiantes en general, y un rendimiento más bajo en el curso de matemática. Esto último puede deberse a diversos factores tanto a nivel de logro del estudiante, pudiendo manifestar bajas expectativas académicas que poseen de ellos mismos, así como un estilo de afrontamiento poco productivo y una falta de creatividad para ser más eficaz y flexible frente a las tareas propias del curso (Lamana \& De la Peña, 2018), o la intervención del docente cuando los alumnos presentan dificultades en el aprendizaje de las matemáticas (Caciá et al., 2012).

Por otro lado, se halló que quienes reciben ayuda de la madre tienen un promedio mayor de teorías fijas que quienes no reciben ayuda de nadie. En este sentido, parece admitirse la idea de que la expectación que tenga la madre en relación al futuro educativo de su hijo es un factor decisivo en su éxito escolar, situándose incluso por encima de los ingresos económicos (Gil, 2011). Con respecto a la relación entre la dimensión de tipo de creencia sobre la inteligencia y el nivel de instrucción, se encontró que los alumnos que prevalecen en su creencia fija reciben ayuda de padres con nivel de instrucción superior incompleta, en comparación de aquellos que no reciben ayuda. Así, Fajardo, Maestre, Felipe, León del Barco y Polo del Río (2017), manifiestan que el rendimiento académico puede verse influido por las expectativas o percepciones que los padres presentan sobre las posibilidades académicas de sus hijos, de manera que es posible que las experiencias educativas de los padres se asocian con el rendimiento académico de sus hijos. Sumado al grado de instrucción de los padres, los escolares que comparten mayor tiempo con el entorno familiar y que la ocupación de la persona encargada de ayudar sea impartir un negocio propio presentan tendencia a una creencia de tipo fija. En consecuencia tal y como argumenta Dweck (1999), estas creencias son consistentes con las que se expresan en su entorno, tanto de los sistemas educativos en los que están inmersos como de sus padres y otros adultos.

Sin embargo, independientemente del estrato económico del que el escolar proviene, las creencias con respecto a la inteligencia y el rendimiento académico no se ven afectados. $\mathrm{Al}$ respecto, Cruz et al. (2014) hallaron una relación débil entre el desempeño escolar y el nivel socio económico, y destaca que el acceso a recursos materiales no define las creencias sobre la inteligencia ni su desempeño académico; sino más bien, el acompañamiento que le brindan en casa al estudiante. Además, es posible que en la medida que la sociedad validada el hecho de que una persona con bajos recursos económicos no pueda desarrollarse académicamente, se da lugar a que en esta investigación no existan diferencias en las creencias sobre la inteligencia en relación al estrato económico (Santín, 2001). 
En lo que respecta a la relación de los factores sociales con el rendimiento académico se observaron diferencias significativas, de modo que las mujeres obtuvieron un rendimiento académico superior al de los hombres en la asignatura de comunicación, coincidiendo con Barahona (2014) quien refiere que las mujeres tenderían a un mejor desempeño académico porque adquieren un comportamiento apropiado, manifestando una mayor responsabilidad y constancia. Además, se observa que aquellos estudiantes que reciben algún apoyo, sea de padre, la madre u otro adulto, tienen un mejor desempeño académico que aquellos que no reciben ayuda de nadie, al menos en el curso de comunicación. Estos resultados son similares a los hallazgos de Lastre, López y Alcázar (2018), quienes señalan que el desempeñoacadémico alto lo obtienen los alumnos que tienen acompañamiento por parte de sus padres. En relación a ello, se encontramos que las familias en las que se ayuda a veces y rara vez a los estudiantes, su desempeño académico más alto que aquellos casos en los que reciben ayuda parental frecuentemente o que no reciben ayuda en lo absoluto en el curso de comunicación. Esto indicaría que la cantidad de veces en que se ayuda al estudiante afecta su desempeño en este curso, siendo necesario moderar la cantidad de veces que el estudiante debe ser ayudado en tal asignatura. A su vez se puede observar que en las familias extensas se tienen mejores notas en comunicación mientras que las familias monoparentales tienen mayores dificultades con el desempeño en matemática. En ese sentido, Cervini, Dari y Quiroz (2014) llegaron a la conclusión de que los educandos de familias con ambos progenitores alcanzan un mejor rendimiento académico al contrario de familias monoparentales, ello se debe según López y Pibaque (2018), a que su situación familiar genera en el estudiante una escasa interacción social, viéndose perjudicado su desempeño académico mediado por el efecto de sus relaciones interpersonales. Barca, Peralbo, Porto, Marcos y Brenlla (2011) respaldan esta particularidad y la asocian a la motivación intrínseca y el aprendizaje autorregulado, como ha sido remarcado en oros estudios (Zimmerman \& Schunk, 2001).

En relación a las creencias acerca de la inteligencia en función al tipo instituciones educativas (privadas o estatales) en que estudian los alumnos, se encontró que aunque en los colegios estatales predomina una creencia de tipo crecencial, su rendimiento es aún bajo. Cabe señalar que en nuestro país los colegios estatales hacen un acompañamiento deficiente del escolar, mientras que las instituciones privadas, motivadas por la competencia institucional, suelen proporcionar mejores oportunidades de preparación académica a los estudiantes. En resumen, tenemos que en relación al análisis de los resultados y nuestro objetivo general, los alumnos con creencia fija tienen un mayor rendimiento en ambos cursos, a comparación de los escolares con creencia de crecimiento, de forma contrapuesta a lo que la Dweck (2017) propone; pero que puede explicarse por la ausencia de situaciones académicas desafiantes que destaquen la influencia del tipo de creencia en el desempeño académico así como por 
un contexto sociofamiliar que no brinda los soportes adecuados, mediados por el grado académico de los padres, su nivel socioeconómico y su acompañamiento académico, así como por la calidad de la enseñanza recibida en las instituciones educativas. Por ello, se puede concluir que existe una predominancia por la creencia de tipo fija como consecuencia de las percepciones del niño sobre la inteligencia, su contexto cultural y la influencia de los padres.

Por todo lo señalado, se recomienda continuar con investigaciones en el campo de la psicología educativa ya que ello favorecen a la educación peruana y por ende a nuestro país que tanto requiere de mejoras basadas en evidencias científicas. En efecto, es fundamental que principalmente en esta rama de la psicología, se promueva la aplicación de estos hallazgos mediante diversos recursos psicológicos, educacionales y psicopedagógicos.

\section{Financiamiento}

El estudio fue autofinanciado.

\section{Conflictos de interés}

Las autoras declaran que no tienen conflictos de interés. 


\section{Referencias}

Abd- El- Fattah, S., \& Yates, G. (2006). Implicit Theory of Intelligence Scale. Testing for factorial invariance and mean structure. Paper presentedo en la Australian Association for Research in Education Annual Conference. https://pdfs.semanticscholar.org/o4cd/374aa46ef5d5of6949fe31c463fi83bf2759.pdf

Álvarez, F. (2012). Diferencias en la calidad de educación e ineficiencia: Un análisis basado en el método de frontera estocástica. Documentos de Trabajo (pp. 1-25). CAF https://scioteca.caf.com/bitstream/handle/123456789/242/diferencias_calidad_educacion_ineficiencia_metodo_frontera_estocastica.pdf

Aparicio, M., Cala, M., \& Adarve, S. (2010). Teorías implícitas de la inteligencia en estudiantes de quinto a séptimo grado de un colegio del municipio de Girón, Santander. II Congreso Internacional de Investigación y Práctica Profesional en Psicología (pp. 312-314). https://www.aacademica.org/ooo-031/417.pdf

Arias, W. L. (2013). Teoría de la Inteligencia: una aproximación neuropsicológica desde el punto de vista de Lev Vigotsky. Cuadernos de Neuropsicología, 7(1), 22-37. http//:doi.org/10.7714/cnps/7.1.201

Arias, W. L. (2014). Estilos de aprendizaje e inteligencia en estudiantes universitarios de Arequipa (Perú). Journal of Learning Styles, 7(14), 88-107. http://learningstyles. uvu.edu/index.php/jls/article/view/201/175

Asociación Peruana de Empresas de Investigación de Mercado (2013). Aspectos metodológicos. http://apeim.com.pe/

Aronson, J., Fried, C. B., \& Good, C. (2002). Reducing the Effects of Stereotype Threat on African American College Students by Shaping Theories of Intelligence. Journal of Experimental Social Psychology, 38(2), 113-125. http://dx.doi.org/10.1006/ jesp.2001.1491

Backhoff, E., Sánchez M., Peón M. \& Andrade, E. (2010). Comprensión lectora y habilidades matemáticas de estudiantes de educación básica en México: 2000-2005. Revista Electrónica de Investigación Educativa, 2(1) 1-15. https://redie.uabc.mx/ redie/article/view/242/738

Barahona, P. (2014). Factores determinantes del rendimiento académico de los estudiantes de la Universidad de Atacama. Estudios Pedagógicos, 40(1), 25-39. http://dx.doi.org/10.4067/So718-07052014000100002 
Barca, L. A., Peralbo, U. M., Porto, R. A., Marcos, M. J. L., y Brenlla, B. J. C. (2011). Metas académicas del alumnado de Educación Secundaria Obligatoria (ESO) y Bachillerato con alto y bajo rendimiento escolar. Revista de Educación, 354, 341-368.

BBC. (2016). Los países de América Latina “con peor rendimiento académico". http:// www.bbc.com/mundo/noticias/2016/02/160210_paises_bajo_rendimiento_educacioniinforme_ocde_bm

Berríos, C. (2018). Docentes y sus creencias sobre la inteligencia: ¿Están en sintonía con las discusiones actuales? Castalia, 4(30), 68-78. https://doi. org/10.25074/o7198051.30.911

Binet. A., \& Simon, T. (1916). The development of intelligence in children. Williams \& Wilkins.

Blackwell, L., Trzesniewski, K. \& Dweck, C. (2007). Implicit theories of intelligence predict achievement across and adolescent transition: A longitudinal study and intervention. Child Development, 78(1), 246-263. https://doi. org/10.1111/j.1467-8624.2007.00995.X

Boullosa, G. (2014). Teorías Implícitas sobre la evaluación de los aprendizajes que poseen los docentes de arquitectura de una universidad privada en Lima. (Tesis para optar por el grado de Magíster en Educación). Pontificia Universidad Católica del Perú, Lima, Perú. http://tesis.pucp.edu.pe/repositorio/bitstream/ handle/20.500.12404/5499/BOULLOSA_RIVAS_GLORIA_TEORIAS_DOCENTES. pdf? sequence $=1 \&$ isAllowed $=\mathrm{y}$

Byrnes, J. (1996). Cognitive development and learning in instructional context. Allyn \& Bacon.

Caciá, D., Reyes, M., Rosales, C., \& Marroquín, E. (2012). Factores que inciden en el rendimiento en matemática de niñas y niños del primer ciclo del nivel de educación primaria en escuelas públicas de Guatemala. https://intercoonecta.aecid. es/Gestin\%2odel\%2oconocimiento/Informe\%2oFinal\%2oInvestigacion\%20 en\%2oMatematicas.pdf

Capital (31 de marzo del 2015). ¿Son realmente los colegios privados mejores que los públicos en Perú? https://capital.pe/actualidad/son-realmente-los-colegios-privados-mejores-que-los-publicos-en-peru-noticia-783304 
Camacho, K. (2011). Teorías implícitas de la inteligencia en Docentes de la Universidad Pontificia Bolivariana. (Tesis para optar por el título de psicología). Universidad Pontificia Bolivariana, Medellín, Colombia. https://repository.upb.edu.co/ bitstream/handle/20.500.11912/1153/digital_20765.pdf?sequence=1\&isAllowed=y

Carpintero, E., Cabezas, D., González, C., \& Fernández, P. (2003). Análisis de las teorías implícitas de la inteligencia en alumnos de educación primaria. EduPsykhé, 2(1), 81-105. https://dialnet.unirioja.es/descarga/articulo/1075778.pdf

Cervini, R., Dari N., \& Quiroz, S. (2014). Estructura familiary rendimiento académico en países de América Latina: Los datos del Segundo Estudio Regional Comparativo y Explicativo. Revista Mexicana de Investigación Educativa, 19(61), 569-597. http:// www.scielo.org.mx/scielo.php?script=sci_arttext\&pid=S1405-66662014000200010

Correo (21 de octubre 2013). Colegios privados versus nacionales. https://diariocorreo. pe/peru/colegios-privados-versus-nacionales-71949/?ref=dcr

Correo (29 de enero de 2019). Años escolar 2019: se terminó calificación del o al 20. https://diariocorreo.pe/edicion/lima/ano-escolar-notas-en-libretas-de-secundaria-seran-letras-y-ya-no-numeros-867559/

Cruz, Z., Medina, J., Vázquez, J., Espinosa, E., \& Antonio, A. (2014). Influencia del nivel socioeconómico en el rendimiento académico de los alumnos del programa educativo de ingeniería industrial en la Universidad Politécnica de Altamira. En G.Nélida, G. Santamaria, L. Almaza (Coords.). Manual de Ciencias Administrativas y Sociales (pp. 24-38). https://dialnet.unirioja.es/servlet/articulo? codigo $=4887110$

Cuenca, R., Carillo, S., De los Rios, C., Reátegui, L., \& Ortiz, G. (2017). La calidady equidad de la educación secundaria en el Perú. Institutos de Estudios Peruanos. http://repositorio.iep.org.pe/bitstream/IEP/923/1/Cuenca-Ricardo_CalidadEquidad-Educacion-Secundaria.pdf

De Castella, K., \& Byrne, D. (2015). My intelligence may be more malleable than yours: the revised implicit theory of intelligence (self-theory) scale is a better predictor of achievement, motivation, and student disengagement. European Journal of Psychology of Education, 30(3), 245-267. https://doi.org/10.1007/s10212-015-0244-y

Dongo, M. (2009). Significado de los factores sociales y culturales en el desarrollo cognitivo. Revista de Investigación en Psicología, 2(2), 227-237. https://doi. org/10.15381/rinvp.v12i2.3767 
Dweck, C. S. (1975). The role of expectations and attributions in the alleviation of learned helplessness. Journal of Personality and Social Psychology, 31, 674-685.

Dweck, C. (1986). Motivational processes affecting learning. American Psychologist, 41, 1040-1048. http://dx.doi.org/10.1037/ooo3-066X.41.10.1040

Dweck, C. S. (1999). Self-theories: Their role in motivation, personality, and development. Essays in social psychology. Psychology Press.

Dweck, C.S. (2002). Messages that motivate: How praise molds students' beliefs, motivation, and performance (In Surprising Ways). En J. Aronson (Ed.), Improving academic achievement. Academic Press.

Dweck, C. S. (2007). La actitud al éxito. Ediciones Vergara.

Dweck, C. S. (2017). Mindset: How you can fulfill your potential. Kindle.

Dweck, C. S., Chiu, C., \& Hong, Y. (1995). Implicit Theories: Elaboration and Extension of the Model. Psychological Inquiry, 6(4), 322-333. https://doi.org/10.1207/ s15327965plio6o4_12

Dweck, C. S., \& Elliott, E. S. (1983). Achievement Motivation. En P. H. Mussen (Gen. Ed.), \& E. M. Hetherington (Ed.), Handbook of Child Psychology Vol. 4, (pp. 643-691). Wiley.

Dweck, C. S., \& Legget, E. (1998). A social cognitive approach to motivation and personality. Psychological Review, 95, 256-273. https://doi.org/10.1037/0033-295X.95.2.256

Dweck, C. S., \& Reppucci, N.D. (1973). Learned helplessness and reinforcement responsibility in children. Journal of Personality and Social Psychology, 25, 109-116.

Elliot, A., Harackiewicz, J., Barron, K. Pintrich, P., \& Thrash, T. (2002). Revision of achievement goal theory: Necessary and Illuminating. Journal of Education Psychology, 94(3), 638-645. https://doi.org/10.1037/oo22-0663.94.3.638

El Comercio (3 de diciembre 2016). Prueba PISA: casi 80\% de estudiantes latinoamericanos tienen bajo nivel en lectura y matemáticas. https://elcomercio.pe/ mundo/prueba-pisa-casi-8o-de-estudiantes-latinoamericanos-tienen-bajo-nivel-en-lectura-y-matematicas-nndc-noticia/?ref=ecr 
Escudero, E., Moguel, A., Zapata, M., \& Muñoz, E. (2010). Comprensión lectora y habilidades matemáticas de estudiantes de educación básica en México: 20002005. Revista Electrónica de Investigación Educativa, 12(1). https://redie.uabc. $\mathrm{mx} / \mathrm{redie} /$ article/view/242/738

Ellis, J. (2005). Aprendizaje Humano. 4ta Edición. Pearson Educación.

Fandiño, Y. J. (2008). Una enseñanza e investigación inteligentes de la inteligencia para el éxito escolar y el éxito en la vida cotidiana. Revista Iberoamericana de Educación, 46(9), 1-12. https://rieoei.org/RIE/article/view/1881

Figueroa C. (2004). Sistemas de evaluación académica. El Salvador.

Flores, G., Contreras L., \& Backhoff, E. (2006). Traducción y adaptación de pruebas: lecciones aprendidas y recomendaciones para países participantes en TIMSS. PISSA y otras comparaciones internacionales. Revista Electrónica de Investigación Educativa, 8, (2). https://redie.uabc.mx/redie/article/view/143/245

Galvis, M., Cala, M., \& Adarve S. (2010). Teorías implícitas de la inteligencia en estudiantes de quinto a séptimo grado de un colegio del Municipio de Girón, Santander. II Congreso Internacional de Investigación y Práctica Profesional en Psicología XVIIJornadas de Investigación Sexto Encuentro de Investigadores en Psicología del MERCOSUR. Recuperado de: https://www.aacademica.org/ooo031/417.pdf. El 23 de octubre del 2019.

García L. (2016). Nivel socioeconómico y rendimiento académico: estudiantes resilientes. (Tesis doctoral en Psicología). Universidad Complutense de Madrid, España. https://eprints.ucm.es/38862/1/T37677.pdf. El 23 de octubre del 2019

Gayón, L. (2010). Relación entre las teorías implícitas de la inteligencia de padres e hijos y rendimiento académico. (Tesis para optar el grado de psicóloga). Universidad Pontificia Bolivariana, Medellín, Colombia. https://repository.upb.edu.co/ bitstream/handle/20.500.11912/826/digital_19151.pdf?sequence=1\&isAllowed=y

Gestión (3 de diciembre del 2016). Perú mejora en prueba PISA 2018, pero sigue último entre los países de la región. https://gestion.pe/peru/peru-mejora-en-prueba-pisa-2018-pero-sigue-ultimo-entre-los-paises-de-la-region-nndc-noticia/?ref=gesr.

Gross-Loh, C. (2019). Don't Let Praise Become a Consolation Prize. [online] The Atlantic. https://www.theatlantic.com/education/archive/2016/12/ how-praise-became-a-consolation-prize/510845/ 
Hernández, R., Fernández, C., \& Baptista, P. (2010). Metodología de la investigación.

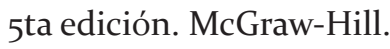

Horn, J. L. (1965). Fluid and crystallized intelligence: A factor analytic study of the structure among primary mental abilities. [Tesis para obtener el grado de Doctor en Psicología] University of Illinois, USA.

Horn, J. L., \& Cattell, R. B. (1966). Refinement and test of the theory of fluid and crystallized general intelligences. Journal of Educational Psychology, 57, 253-270.

Horn, J. L., \& Cattell, R. B. (1967). Age differences in fluid and crystallized intelligence. Acta Psychologica, 26, 107-129.

Hoyos R. (2016). Niveles socioeconómicos y motivación en la elección de la carrera profesional en estudiantes preuniversitarios. (Tesis para optar el grado de Maestra en Psicología). Universidad San Martín de Porres, Lima, Perú. http://www.repositorioacademico.usmp.edu.pe/bitstream/usmp/2320/1/hoyos_drg.pdf

King, R. B. (2019). Mindsets are contagious: The social contagion of implicit theories of intelligence among classmates. British Journal of Educational Psychology. 9o(2), 349-363. https://doi.org/10.1111/bjep.12285

Krakovsky, M. (2019). The Effort Effect: According to a Stanford psychologist, you'll reach new heights if you learn to embrace the occasional tumble. https://stanfordmag.org/contents/the-effort-effect

Kurtz-Costes, B., McCall, R.J., Kinlaw, C.R., Wiesen, C.A., \& Joyner, M.H. (2005). What does it mean to be smart? The development of children's beliefs about intelligence in Germany and the United States. Applied Developmental Psychology, 26, 217-233. http://dx.doi.org/10.1016/j.appdev.2004.12.005

Lamana, M., \& De la Peña, C. (2018). Rendimiento académico en matemáticas. Relación con creatividad y estilos de afrontamiento. Revista Mexicana de Investigación Educativa, 23(79), 1075-1092. http://www.scielo.org.mx/pdf/rmie/v23n79/14056666-rmie-23-79-1075.pdf

Lammel A., \& Guillén E. (2011). Las concepciones implícitas de la inteligencia contextos culturales. Diferentes (México y Francia). Revista Electrónica de Psicología Iztacala, 14(2) 15-19. http://www.medigraphic.com/pdfs/epsicologia/epi-2011/ epin12b.pdf 
Lastre, K., López, L., \& Alcázar, C. (2018). Relación entre apoyo familiary el rendimiento académico en estudiantes colombianos de educación primaria. Psicogente, 21(39), 102-115. http://www.scielo.org.co/pdf/psico/v21n39/o124-0137-psico-21-39-00102. pdf

Linares A. (2008). Desarrollo Cognitivo: Master en paidopsiquiatría. Módulo 1: Las teorías de Piaget y Vygotsky. Universidad Autónoma de Barcelona. http://www. paidopsiquiatria.cat/files/teorias_desarrollo_cognitivo_o.pdf

López, P., \& Pibaque, M. (2018). Familias monoparentales y el desarrollo social en los adolescentes. Revista Científica Dominio de las Ciencias, 4(3), 152-162. 10.23857/ dc.v4i3.799

Macías, M. A. (2002). Las múltiples inteligencias. Psicología desde El Caribe, 10, 27-38. https://www.redalyc.org/pdf/213/21301003.pdf

Makuc, M. (2011). Teorías implícitas sobre comprensión textual y la competencia lectora de estudiantes de primer año de la Universidad de Magallanes. Revista Signos, 48(87), 29-53. https://www.redalyc.org/pdf/1570/157036980oo2.pdf

Marchago, J. (1989). El profesor y el autoconcepto de los alumnos. Escuela Española.

Ministerio de Educación del Perú (2017). Currículo Nacional de la Educación Básica. http://www.minedu.gob.pe/curriculo/pdf/consulta-virtual-del-curriculo-nacional.pdf

Montero, I., \& León, O. (2007). A guide for naming research studies in Psychology. International Journal of Clinical and Health Psychology, 7, 847-862.

Muñiz, J., \& Hambleton. (1996). Directrices para la traducción y adaptación de test. Papeles de Psicólogo, 66. http://www.papelesdelpsicologo.es/resumen?pii=737

Nicholls, J. (1983) Conceptions of ability and achievement motivation: a theory and its implications for education. En S. Paris, G. Olsen, H., Stevenson (Eds.), Learning and motivation in the classroom. Erlbaum.

Noack, E. M. (2018). Uso de Técnicas de mentalidad de crecimiento y su impacto en el rendimiento académico de los estudiantes de grado uno de un colegio privado de la ciudad de Guatemala. (Tesis de grado) http://recursosbiblio.url.edu.gt/ tesiseortiz/2018/o5/83/Montenegro-Erika.pdf 
OCDE (2013). Education at a Glance 2013 OECD indicators. http://www.oecd.org/ education/Panorama\%2ode\%2ola\%2oeducacion\%202013.pdf

Olmeda, L. (2016). Nivel socioeconómico y rendimiento académico: Estudiantes resilientes. [Tesis para optar por el grado de Doctora en Psicología]. Universidad Complutense de Madrid, España. https://eprints.ucm.es/38862/1/T37677.pdf

Peña, A. (2015). Ganadores y perdedores: fecha de nacimiento, edad relativa en la escuela, y los resultados en la infancia y la edad adulta. http://ntrzacatecas.com/2015/11/15/ influye-la-edad-de-alumnos-en-su-rendimiento-escolar-revela-estudio/

Romagnoli, C., \& Cortese, I. (2015). ¿Cómo la familia influye en el aprendizaje y rendimiento escolar? Valoras. www.valoras.uc.cl

Rodríguez, J. (2015). El rendimiento escolar y la intervención del trabajo social. Universidad de Cuenca.

Sánchez, P., Valdés, A., Reyes, N., \& Martínez, E. (2010). Participación de padres de estudiantes de educación primaria en la educación de sus hijos en México. Liberabit, 16(1), 71-80.

Santín D. (2001). Influencia de los factores socioeconómicas en el rendimiento escolar internacional: hacia la igualdad de oportunidades educativas. http://eprints. ucm.es/6725/1/o101.pdf

Spearman, C. (1904). "General intelligence”, Objectively determined and measured. American Journal of Psychology, 15, 201-293. https://doi.org/10.2307/1412107

Soto, M., Da Cuña, I., Lantarón, M., \& Labajos, T. (2015) Influencia de las variables sociodemográficas y socioeducativas en el rendimiento académico de alumnos del Grado en Fisioterapia. FEM: Revista de la Fundación Educación Médica, 18(6), 397-404. https://doi.org/10.4321/s2014-98322015000700007

Stromso, H., \& Braten, I. (2004). Epistemological beliefs and implicit theories of intelligence as predictors of achievement goals. Contemporary Educational Psychology, 29, 371-388. https://doi.org/10.1016/j.cedpsych.2003.10.001

Sulmont, L. (2018). Implementación sistemática en las escuelas de las expectativas de aprendizaje y enseñanza para alumnos y docentes. AD MESTRO, 1(3), 14-17. 
Tarbetsky, A., Collie. R., \& Martin, A. (2016). The role of implicit theories of intelligence and ability in predicting achievement from indigenous Australian students. Contemporary Educational Psychology, 47, 61-71. https://doi.org/10.1016/j. cedpsych.2016.01.002

Tomás, J., \& Almenara, J. (2008). Desarrollo cognitivo: Las teorías de Piaget y de Vygotsky. Master en Paidopsiquiatria. Universidad Autónoma de Barcelona. http://www.paidopsiquiatria.cat/files/teorias_desarrollo_cognitivo_o.pdf

UNESCO (2015). Informe de resultados TERCE: Tercer estudio regional comparativo y explicativo. Logros de aprendizaje. https://unesdoc.unesco.org/ark:/48223/ pfoooo243532

Universidad de Internacional de Valencia. (2015). Influencia de los factores sociales y familiares en el bajo rendimiento. https://www.universidadviu.es/influencia-de-los-factores-sociales-y-familiares-en-el-bajo-rendimiento-en-el-aprendizaje/

Vélez, E., Schiefelbein, E., \& Valenzuela, J. (1994). Factores que afectan el rendimiento académico en la educación primaria. Revisión de la literatura de América Latina y el Caribe. Revista Latinoamericana de Innovación de América Latina, 17, 29-53. http://disde.minedu.gob.pe/bitstream/handle/123456789/4317/ Factores\%2oque\%2oafectan\%2oel\%2orendimiento\%2oacad\%C3\%A9mico\%20 en\%2ola\%2oeducaci $\% \mathrm{C}_{3} \% \mathrm{~B}_{3}$ \%2oprimaria\%2orevisi $\% \mathrm{C}_{3} \% \mathrm{~B} 3$ n\%2ode $\% 20$ la\%2oLiteratura\%2ode\%2oAm\%C3\%Agrica\%2oLatina\%2oy\%2oEl\%2oCaribe. pdf? sequence $=1 \&$ isAllowed $=\mathrm{y}$

Vera, F., \& Vera, O. (2013). Evaluación del nivel socioeconómico: presentación de una escala adaptada en una población de Lambayeque. Revista del Cuerpo Médico Hospital Almanzor Aguinaga Asenjo, 6(1). https://dialnet.unirioja.es/servlet/ articulo? codigo $=4262712$

Villamizar, G. (2011). Teorías implícitas de la inteligencia en el ámbito pedagógico. Revista Psicogente, 14(26), 321-335. http://oaji.net/articles/2017/1787-1485448348. pdf

Wang, Q., \& Fei-YinNg F. (2012). Chinese students' implicit theories of intelligence and school performance: Implications for their approach to schoolwork. Personality and Individual Differences, 52(8), 930-935. https://doi.org/10.1016/j.paid.2012.01.024

Woolfolk, A. (2010). Psicología educativa. Pearson Educación. 
Yeager, D.S., \& Dweck, C.S. (2012). Mindsets that promote resilience: When students believe that personal characteristics can be developed. Educational Psychologist, 47(4), 302-3014. https://doi.org/10.108o/00461520.2012.722805

Zapata, M. (2017). Teorías y modelos sobre los aprendizajes en entornos conectados y ubicuos. Bases para un nuevo modelo teórico a partir de una visión crítica del "conectivismo". Education in the knowledge Society, 16(1), 69-102. https://doi. org/10.14201/eks201516169102

Zegarra, C. D. (2013). Aspiraciones educativa y ocupacional de jóvenes: un estudio mixto sobre el rol del nivel socioeconómico y la percepción de barreras. [Tesis para optar por el título de Psicología con mención en Psicología Educacional]. Pontificia Universidad Católica del Perú, Lima, Perú. http://tesis.pucp.edu.pe/ repositorio/handle/20.500.12404/4840

Zimmerman, B. J., \& Schunk, D. H. (2001) Self-Regulated Learning and Academic Achievement: Theoretical Perspectives. Lawrence Erlbaum Associates.

Recibido: 25 de junio de 2020

Revisado: 8 de octubre de 2020

Aceptado: 12 de marzo de 2021 